ESAIM: M2AN 50 (2016) 565-591

DOI: $10.1051 / \mathrm{m} 2 \mathrm{an} / 2015056$
ESAIM: Mathematical Modelling and Numerical Analysis

www.esaim-m2an.org

\title{
ANALYSIS OF THE PENALIZED 3D VARIABLE VISCOSITY STOKES EQUATIONS COUPLED TO DIFFUSION AND TRANSPORT
}

\author{
Robin Chatelin ${ }^{1}$, David Sanchez ${ }^{2}$ and Philippe PonceT ${ }^{3}$
}

\begin{abstract}
The analysis of the penalized Stokes problem, in its variable viscosity formulation, coupled to convection-diffusion equations is presented in this article. It models the interaction between a highly viscous fluid with variable viscosity and immersed moving and deformable obstacles. Indeed, while it is quite common to couple Poisson equations to diffusion-transport equations in plasma physics or fluid dynamics in vorticity formulations, the study of complex fluids requires to consider together the Stokes problem in complex moving geometry and convection-diffusion equations. The main result of this paper shows the existence and the uniqueness of the solution to this equations system with regularity estimates. Then we show that the solution to the penalized problem weakly converges toward the solution to the physical problem. Numerical simulations of fluid mechanics computations in this context are also presented in order to illustrate the practical aspects of such models: lung cells and their surrounding heterogeneous fluid, and porous media flows. Among the main original aspects in the present study, one can highlight the non linear dynamics induced by the coupling, and the tracking of the time-dependence of the domain.
\end{abstract}

Mathematics Subject Classification. 35Q30, 76D03, 76D07, 65M25, 68U20, 76Z05, 92B05.

Received January 29, 2014. Revised May 5, 2015.

Published online March 14, 2016.

\section{INTRODUCTION}

The dynamics of a three dimensional flow is usually modeled by the Navier-Stokes equations, coming from the conservation of the fluid's mass and momentum. The Reynolds number is a dimensionless ratio of inertial forces to viscous forces. When it is very small the convection terms in the conservation of momentum equation vanish and the Navier-Stokes equations are reduced to the Stokes equations. In the literature many authors analyzed the solutions to these equations and implemented their numerical resolution, but a lot of work remains, particularly when the fluid is interacting with immersed obstacles and when its composition is not homogeneous.

\footnotetext{
Keywords and phrases. Stokes equations, moving geometry, variable viscosity flows, porous media flows, biomathematics.

1 Université de Lyon, ENISE, LTDS UMR CNRS 5513, 58 rue Jean Parot, 42023 Saint-Étienne cedex 02, France. robin.chatelin@enise.fr

2 Toulouse Mathematics Institute, UMR CNRS 5219, Team MIP, INSA, GMM 135 avenue de Rangueil, 31077 Toulouse, France.

3 LMAP, UMR CNRS 5142, IPRA, UPPA, avenue de l'Université, BP 1155, 64013 Pau, France.
} 
Mathematical models for numerical computations of fluid flows, interacting with solid or porous obstacles, can be divided in two families [23]. In the first category the mesh has to follow precisely the moving objects and boundary conditions can be straightly applied to these boundaries $[22,24]$. It requires the mesh to move with Arbitrary Lagrangian Eulerian techniques [32] and to often remesh the domain: this is very difficult to handle numerically for large problems [27], particularly in 3D. On the other hand it is possible to consider a single mesh and to treat moving obstacles through their characteristic functions. Thus the precise position of the solid interface is not required. This class of problems are referred as fictitious domain methods or penalty methods. Different penalty can be implemented in various ways inside obstacles volume using a small penalty parameter $\varepsilon$ to force a prescribed solid velocity [5,14] or imposing a rigid motion by penalizing the strain [29] with an adequate variational formulation and Lagrange multipliers. For all these methods an extra term is added in the conservation of momentum equation.

In this work we focus on the $L^{2}$ volumetric penalty of the velocity [5], since the numerical applications focus on large 3D problems. With this large number of degrees of freedom the assembled matrix condition number is very high and deteriorated by penalty terms [33] and computational techniques to avoid assembling are easier to implement with this approach. Hence the answer to two questions is requested. Does the solution to the penalized problem exist and is it unique? Does this solution converge toward the solution to the physical problem when the penalty parameter tends to zero?

Existence and uniqueness of solutions to the penalized Navier-Stokes equations with constant viscosity and regularity estimates were developed in [5] and a precise analysis of the boundary layer gave a good agreement with numerical experiments in [9]. Similar results for the Stokes flow with variable viscosity was also investigated in [4] but only for a linear dynamics. A two-ways fluid-structure interaction was also analyzed in [7] for rigid obstacles.

The present work brings the analysis of the penalized Stokes problem or Stokes/Brinkman problem when the viscosity depends on the fluid velocity through the convection-diffusion of a certain mass fraction. This situation arises for non homogeneous creeping flows, in biological situations for example, when the viscosity is directly influenced by a certain component (miscible or not) of the fluid. This model is particularly relevant when a microscopic model of this agent evolution is not available: this can be a consequence of the lack of experimental data (very small scale phenomena) or too complex dynamics (hundreds of proteins interactions in biological systems for example). This coupling between viscosity and velocity induces a non linear time dependent dynamics.

The equation system under consideration in this work is similar to the non-homogeneous Navier-Stokes system or the penalized Navier-Stokes system [8], but in this article we consider a non-homogeneous fluid interacting with immersed obstacles. The fluid satisfies the Stokes problem, which is stationary, but the obstacles are moving and deforming. This introduces an implicit time dependency which must be tracked in the computations using 4-dimension Sobolev spaces. In the following demonstrations, we use a Banach fixed-point theorem to prove the existence of solutions. The main point lies in the proof of time-dependent estimates to obtain at first local in time existence. Unlike [8] we recover the time-regularity from the convection-diffusion of the mass fraction and not from Navier-Stokes equations where regularity comes from the time derivation. Then this time-dependency needs to be tracked in the Stokes problem to finally obtain existence and uniqueness of the solutions with $\varepsilon$-independent bounds, which allow to prove existence and uniqueness of the solutions outside the obstacles.

3D numerical computations are also presented to illustrate two flow configurations governed by this equation set. The first one is the computation of a passive tracer inside a porous media geometry at the scale of the pores. The second numerical example is the computation of a non-homogeneous mucus flow around epithelium cells, a problem of high interest for the study of lung pathologies such as cystic fibrosis.

After introducing the model, the main result is presented: existence and uniqueness is first given with regularity estimates with fixed penalty parameter. Then we show that the limit solution, when this parameter tends to zero, fulfills the good equation set. This justifies the choice of the penalized system for our numerical approach. The demonstration of this result follows. Finally the numerical illustrations are presented. 


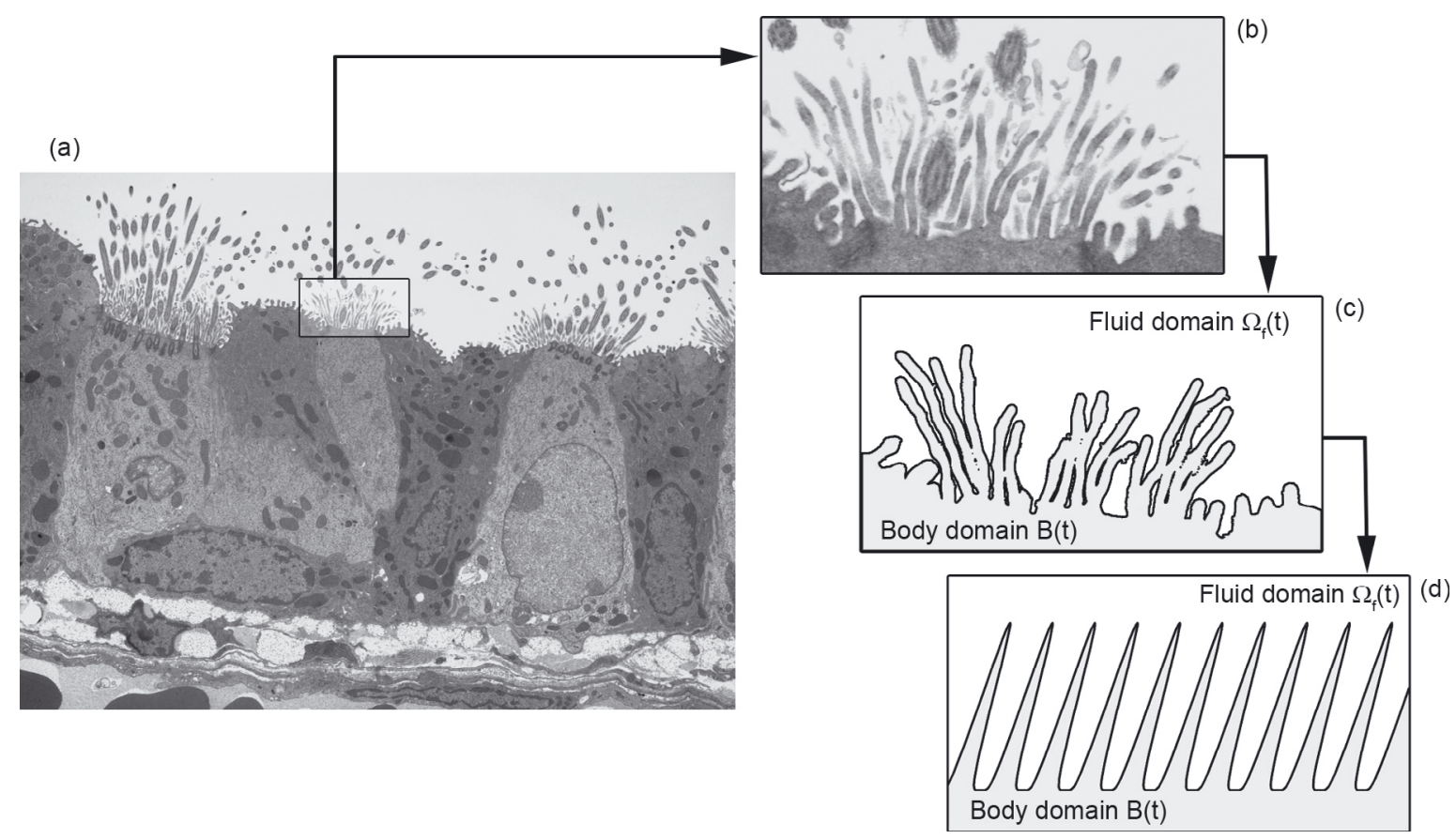

FiguRE 1. The moving domain $B(t)$ models the fixed bronchial wall of the lung and the moving cilia. In the following we consider the model (d) where the boundary $\partial B(t)$ is regular.

\section{Problem SETUP AND MODELING}

When a fluid is very viscous the diffusion dominates the convection and corresponding terms in Navier-Stokes equations vanish. The fluid velocity is then the solution to an inviscid Stokes problem. In this context we analyze a variable viscosity flow in a 3D domain $\Omega$ (precised in the next section) with immersed obstacles. This work concerns a one-way solid-fluid coupling, it means only the force exerted by the solid on the fluid is considered. The inverse mechanism is not treated in the following. Hence the solid velocity $\bar{u}$ is a time dependent data imposed inside the solid domain (which also depends on $t$ ) whose evolution is also considered as a data. It means that the solid domain, called $B(t)$ in the following and that models the lung bronchial wall covered by cilia (see Fig. 1), evolves with its own velocity $\bar{u}$.

To model this variable viscosity, it is assumed to depend on a certain mass fraction $\alpha$, solution to a convectiondiffusion equation (with a dominating convection). This model is justified when the viscosity straightly depends on an immiscible agent in the fluid (for example chemicals, proteins, polymers,... in water). In this case the viscosity is non-homogeneous and the diffusion term in the conservation of momentum equation is not reduced to a Laplacian. Hence the viscosity is assumed to be a function of the mass fraction of this agent: $\mu=\Phi(\alpha)$. Thus the viscosity is bounded: $\mu_{0} \leq \Phi(\alpha) \leq \mu_{1}, \mu=\mu_{0}$ (equal to water's viscosity) if no agent is present, $\mu=\mu_{1}$ if the fluid is saturated.

This modeling is a good alternative to rheological constitutive laws as they do not always exist for complex fluids and rheological results from the literature are not always agreeing. It is also suitable when two phases of the fluid can be observed: a high concentration zone, and a lower one; but when the interface cannot be tracked precisely as this concentration varies continuously (for biological fluids like the mucus this agent is a protein: the mucin, diluted in water, which gives a gel aspect). Finally this model can be used when a microscopic model of the agent evolution is not available, particularly for biological interactions at small scales, when in-vivo experiments are too invasive to build a relevant model. 
With these assumptions, the problem we are interested in writes:

$$
\left\{\begin{array}{lr}
u=\bar{u} & \text { inside solid, } \\
-\operatorname{div}(2 \mu D(u))=f-\nabla p & \text { inside fluid, } \\
\operatorname{div} u=0 & \text { inside fluid, } \\
\partial_{t} \alpha+u \cdot \nabla \alpha-\eta \Delta \alpha=0 & \text { everywhere in computational domain, } \\
\mu=\Phi(\alpha) & \text { everywhere in computational domain, }
\end{array}\right.
$$

where $p$ is the pressure, $\mu$ is the fluid viscosity, $f$ are the external forces and $D(u)=\left(\nabla u+(\nabla u)^{T}\right) / 2$ is the strain tensor. With initial and boundary conditions yet to be set.

To consider the solid-fluid interaction a penalty term is added in the Stokes conservation of momentum equation to force the velocity $u$ to be equal to a prescribed solid velocity $\bar{u}$ in the solid region $B(t)$. In this way the conservation of momentum equation is extended in the whole domain. This transforms the previous problem into the Penalized Stokes problem or the Stokes-Brinkman problem coupled to a convection-diffusion equation generating a non-linear dynamics:

$$
\begin{cases}-\operatorname{div}\left(2 \mu^{\varepsilon} D\left(u^{\varepsilon}\right)\right)+\frac{\mathbf{1}_{B(t)}}{\varepsilon}\left(u^{\varepsilon}-\bar{u}\right)=f-\nabla p^{\varepsilon} & \text { in } \Omega, \\ \operatorname{div} u^{\varepsilon}=0 & \text { in } \Omega, \\ \partial_{t} \alpha^{\varepsilon}+u^{\varepsilon} \cdot \nabla \alpha^{\varepsilon}-\eta \Delta \alpha^{\varepsilon}=0 & \text { in } \Omega, \\ \mu^{\varepsilon}=\Phi\left(\alpha^{\varepsilon}\right) & \text { in } \Omega,\end{cases}
$$

with $\mathbf{1}_{B(t)}$ the characteristic function of the solid domain and $\varepsilon$ the penalty parameter.

Remark 2.1. In problems (2.1) and (2.2) the convection-diffusion equation is defined in the whole computational domain: no distinction is made between the fluid and the solid domains (hence the initial condition $\alpha_{0}$ will also be defined in the whole computational domain). It means that a weak coupling is assumed, because the quantity $\alpha$ inside the body is not involved in the relation $u=\bar{u}$. Such a convection-diffusion model allows a small diffusion of $\alpha$ between the solid and the fluid, which can be neglected in practice when studying the global dynamics of the system (this is due to the small values of $\eta$ and diffusion effects compared to transport for the targeted applications). Eventually, from a numerical point of view this assumption does not influence the computations since the penalty term dominates considerably the viscous term.

For numerical applications, we work in a bounded computational domain and we are interested in three types of boundary conditions:

- Dirichlet boundary conditions on $\Gamma_{0}$;

- periodic boundary conditions:

- a combination of Neumann boundary conditions for tangential components of velocity and Dirichlet boundary condition for normal component of velocity on $\Gamma_{L}$. This condition is a simplification of the case of a free surface boundary condition: $(2 \mu D(u)-p I) \cdot \nu=\varsigma \kappa \nu$, with $\varsigma$ the surface tension at the interface and $\kappa$ the curvature of this interface. In fact assuming a flat stationary interface we get on $\Gamma_{L}$ : $\varsigma \kappa=0$ and $u_{3}=0$. Then, denoting $\nu$ the outward normal to this boundary and projecting this free surface condition on $\nu^{\perp}$, one gets $\partial_{1} u_{3}=\partial_{2} u_{3}=0$, which is exactly the desired combination.

In the following we consider a regular parallelepiped computational box with periodic boundary conditions with respect to $x$ and $y$ directions, homogeneous Dirichlet boundary conditions at the bottom of computational box and the third Neumann/Dirichlet combination at the top. These conditions are applied for the second numerical application.

In the next section, before the presentation and the demonstration of well-posedness, additional definitions are introduced to complete this problem definition. 


\section{AnAlysis AND WeLL-POSEDNESS}

Let $T>0$. Let $\Gamma=\left[0, L_{1}\right] \times\left[0, L_{2}\right]$ and $\Omega=\Gamma \times[0, L]$ the domain of study. We denote by $\Gamma_{0}=\Gamma \times\{0\}$ and $\Gamma_{L}=\Gamma \times\{L\}$ the lower and upper boundaries of $\Omega$.

Since the domain $B$ depends on the time parameter $t \in[0, T]$, we need to track this dependency in the following. We let

$$
\begin{aligned}
& O=[0, T] \times \Omega \text { the whole domain in time and space coordinates, } \\
& O_{p}=\{(t, x) \in[0, T] \times \Omega, \forall t \in[0, T], x \in B(t)\} \text { the penalized domain, } \\
& O_{p}^{c}=\{(t, x) \in[0, T] \times \Omega, \forall t \in[0, T], x \in \Omega \backslash \bar{B}(t)\}=O \backslash \bar{O}_{p}, \\
& \Sigma=\{(t, x) \in[0, T] \times \Omega, \forall t \in[0, T], x \in \partial B(t) \backslash \partial \Omega\} \text { the inner boundary of } O_{p} .
\end{aligned}
$$

The problem (2.1) rewrites in these spaces:

$$
\left\{\begin{array}{l}
u=\bar{u} \text { in } O_{p} \\
-\operatorname{div}(2 \mu D(u))=f-\nabla p \text { in } O_{p}^{c} \\
\operatorname{div} u=0 \text { in } O \\
\partial_{t} \alpha+u \cdot \nabla \alpha-\eta \Delta \alpha=0 \text { in } O \\
\mu=\Phi(\alpha) \text { in } O
\end{array}\right.
$$

with the initial and boundary conditions described previously:

$$
\left\{\begin{array}{l}
u=0 \text { on }[0, T] \times \Gamma_{0} \\
u=\bar{u} \text { on } \Sigma, \\
u \cdot \nu=\frac{\partial u_{1}}{\partial \nu}=\frac{\partial u_{2}}{\partial \nu}=0 \quad \text { on }[0, T] \times \Gamma_{L}, \\
u \text { is periodic on }[0, T] \times \partial \Gamma \times[0, L], \\
\frac{\partial \alpha}{\partial \nu}=0 \text { on }[0, T] \times\left(\Gamma_{0} \cup \Gamma_{L}\right), \\
\alpha \text { is periodic on }[0, T] \times \partial \Gamma \times[0, L], \\
\alpha(0, \cdot)=\alpha_{0} \text { in } \Omega,
\end{array}\right.
$$

where $\eta>0$ is the diffusion coefficient, $\Phi \in \mathrm{W}^{3, \infty}(\mathbb{R})$ and there exists $0<\mu_{0}$ a real such that for all $\alpha \in \mathbb{R}$ $\mu_{0} \leq \phi(\alpha)$.

According to the modeling, we rewrite the penalized problem:

$$
\begin{cases}-\operatorname{div}\left(2 \mu^{\varepsilon} D\left(u^{\varepsilon}\right)\right)+\frac{\mathbf{1}_{B(t)}}{\varepsilon}\left(u^{\varepsilon}-\bar{u}\right)=f-\nabla p^{\varepsilon} & \text { in } O \\ \operatorname{div} u^{\varepsilon}=0 & \text { in } O \\ \partial_{t} \alpha^{\varepsilon}+u^{\varepsilon} \cdot \nabla \alpha^{\varepsilon}-\eta \Delta \alpha^{\varepsilon}=0 & \text { in } O \\ \mu^{\varepsilon}=\Phi\left(\alpha^{\varepsilon}\right) & \text { in } O\end{cases}
$$

with its initial and boundary conditions coming straightly from the equation set (3.2).

$$
\begin{cases}u^{\varepsilon}=0 & \text { on }[0, T] \times \Gamma_{0}, \\ u^{\varepsilon} \cdot \nu=\frac{\partial u_{1}^{\varepsilon}}{\partial \nu}=\frac{\partial u_{2}^{\varepsilon}}{\partial \nu}=0 & \text { on }[0, T] \times \Gamma_{L}, \\ u^{\varepsilon} \text { is periodic } & \text { on }[0, T] \times \partial \Gamma \times[0, L], \\ \frac{\partial \alpha^{\varepsilon}}{\partial \nu}=0 & \text { on }[0, T] \times\left(\Gamma_{0} \cup \Gamma_{L}\right), \\ \alpha^{\varepsilon} \text { is periodic } & \text { on }[0, T] \times \partial \Gamma \times[0, L], \\ \alpha^{\varepsilon}(0, \cdot)=\alpha_{0} & \text { in } \Omega .\end{cases}
$$


Finally the following functional spaces are introduced:

Definition 3.1. We let $\mathcal{F}(\Omega ; X)$ the set of functions defined on $\Omega$ with value in $X$ and

$$
\begin{aligned}
& \mathrm{L}_{0}^{2}(\Omega)=\left\{v \in \mathrm{L}^{2}(\Omega), \int_{\Omega} v \mathrm{~d} x=0\right\} \\
& V(\Omega)=\left\{v \in \mathrm{H}^{1}(\Omega), \operatorname{div} v=0 \text { on } \Omega, u=0 \text { on } \Gamma_{0}\right. \text {, } \\
& \left.u \cdot \nu=0 \text { on } \Gamma_{L}, u \text { has periodic conditions on } \partial \Gamma \times[0, L]\right\}
\end{aligned}
$$

with the classical $\mathrm{H}^{1}$-norm. Thanks to the generalized Poincaré's inequality (see [8] Sect. III.6), there exist two constants depending only on $\Omega$ such that

$$
c_{1}\|\nabla v\|_{\mathrm{L}^{2}(\Omega)} \leq\|v\|_{\mathrm{H}^{1}(\Omega)} \leq c_{2}\|\nabla v\|_{\mathrm{L}^{2}(\Omega)} .
$$

\subsection{Main results}

The following existence and uniqueness results require some regularity assumptions on the obstacle's time evolution (H1)-(H2) and on the velocity $\bar{u}$ inside the obstacle (H3):

\section{Hypothesis 1. (H1)}

- The domain $B$ is an open set in $\Omega$ having the uniform $\mathcal{C}^{2}$-regularity property (see [2] Sect. 4 for the definition) up to the boundary of $\Omega$ in order to obtain a periodic smooth domain,

- $\exists\left(l, l^{\prime}\right) \in\left(\mathbb{R}^{+}\right)^{2}$ such that $0<l<l^{\prime}<L$ and $\Gamma \times[0, l] \subset B \subset \Gamma \times\left[0, l^{\prime}\right]$, i.e. we assume that the bronchial wall is the fixed domain $\Gamma \times[0, l]$ to which cilia are attached and that the cilia never reach the top of the domain $\Omega$,

- $\Omega \backslash \bar{B}$ is an open set in $\Omega$ having the uniform $\mathcal{C}^{2}$-regularity property,

- $\Omega \backslash \bar{B}$ is simply connected.

Remark 3.2. the inner boundary $\sigma=\partial B \backslash \partial \Omega$ of $B$ is smooth and may be smoothly periodically extended. It moreover splits $\Omega$ in two regular open sets.

\section{Hypothesis 2. (H2)}

- There exists a function $\Psi \in \mathcal{C}^{0}\left([0, T] ; \mathcal{C}^{2}(\Omega)\right)$ such that for all $t \in[0, T] \Psi(t)_{\mid[0, l] \times \Gamma}=I d_{[0, L] \times \Gamma}$, $\Psi(t)\left(\left[0, l^{\prime}\right] \times \Gamma\right) \subset\left[0, l^{\prime}\right] \times \Gamma$ and $\Psi(t)$ has periodic boundary conditions on $[0, L] \times \partial \Gamma$,

- $\forall t \in[0, T] \Psi(t)$ is a $\mathcal{C}^{2}$-diffeomorphism on $\Omega$.

- $\forall t \in[0, T] B(t)=\Psi(t, B)$.

Remark 3.3. we assume that the bronchial wall never moves and that the cilia never reaches the top of the domain. We also have $\Omega \backslash \bar{B}(t)=\Psi(t, \Omega \backslash \bar{B})$.

\section{Hypothesis 3. (H3)}

- $\bar{u} \in \mathcal{C}^{0}\left([0, T] ; H^{2}(B(\cdot))\right)$

- $\forall t \in[0, T], \operatorname{div} \bar{u}(t, \cdot)=0$ in $B(t)$,

- $\forall t \in[0, T] \bar{u}(t, \cdot)$ fulfills

$$
\forall t \in[0, T] \quad \bar{u}(t, \cdot)_{\mid \Gamma_{0}}=0,
$$

- $\forall t \in[0, T] \bar{u}(t, \cdot)$ has periodic boundary conditions on $\partial B(t) \cap(\partial \Gamma \times[0, L])$.

Definition 3.4. $\left(u^{\varepsilon}, p^{\varepsilon}, \alpha^{\varepsilon}\right)$ is a weak solution to (3.3)-(3.4) if it fulfills

- $u^{\varepsilon} \in \mathrm{L}^{q}(0, T ; V(\Omega)), p^{\varepsilon} \in \mathrm{L}^{q}\left(0, T ; \mathrm{L}_{0}^{2}(\Omega)\right)$ with $2 \leq q \leq+\infty, \alpha^{\varepsilon} \in \mathrm{L}^{\infty}\left(0, T ; \mathrm{H}^{2}(\Omega)\right) \cap L^{2}\left(0, T ; \mathrm{H}^{3}(\Omega) \cap\right.$ $\mathrm{H}^{1}\left(0, T ; \mathrm{H}^{1}(\Omega)\right)$; 
- for all $v \in \mathcal{C}_{c}(0, T ; V(\Omega))$;

$$
\iint_{(0, T) \times \Omega} 2 \mu^{\varepsilon} D\left(u^{\varepsilon}\right): D(v) \mathrm{d} x \mathrm{~d} t+\frac{1}{\varepsilon} \iint_{O_{p}}\left(u^{\varepsilon}-\bar{u}\right) \cdot v \mathrm{~d} x \mathrm{~d} t=\iint_{(0, T) \times \Omega} f \cdot v \mathrm{~d} x \mathrm{~d} t,
$$

- $\alpha^{\varepsilon}$ is the solution to

$$
\left\{\begin{array}{l}
\partial_{t} \alpha^{\varepsilon}+u^{\varepsilon} \cdot \nabla \alpha^{\varepsilon}-\eta \Delta \alpha^{\varepsilon}=0 \text { in } \Omega, \\
\frac{\partial \alpha^{\varepsilon}}{\partial \nu}=0 \text { on } \Gamma_{0} \cup \Gamma_{L}, \\
\alpha^{\varepsilon} \text { has periodic conditions on } \partial \Gamma \times[0, L], \\
\alpha^{\varepsilon}(0, \cdot)=\alpha_{0} \text { in } \Omega,
\end{array}\right.
$$

- $\mu^{\varepsilon}=\Phi\left(\alpha^{\varepsilon}\right)$.

Theorem 3.5. Let $0<\mu_{0}$ and $\varepsilon>0$. Let $\alpha_{0} \in H^{2}(\Omega)$ and $\Phi \in W^{2, \infty}(\mathbb{R})$ such that $\forall x \in \mathbb{R}, \mu_{0} \leq \Phi(x)$. We moreover assume (H1), (H2), (H3) and that for all $T>0, f \in L^{\infty}\left(0, T ; L^{2}(\Omega)\right)$. There exists then a unique weak solution $\left(u^{\varepsilon}, p^{\varepsilon}, \alpha^{\varepsilon}\right)$ to (3.3) such that

$$
\left\{\begin{array}{l}
\alpha^{\varepsilon} \in L^{\infty}\left(0, T ; H^{2}(\Omega)\right) \cap L^{2}\left(0, T ; H^{3}(\Omega)\right) \cap H^{1}\left(0, T ; H^{1}(\Omega)\right), \\
u^{\varepsilon} \in L^{q}(0, T ; V(\Omega)) \quad \text { for all } 2 \leq q \leq+\infty \\
p^{\varepsilon} \in L^{q}\left(0, T ; L_{0}^{2}(\Omega)\right) \quad \text { for all } 2 \leq q \leq+\infty
\end{array}\right.
$$

Moreover there exists some constants independent from $\varepsilon$ such that $u^{\varepsilon}$ fulfills

$$
\begin{aligned}
& \mu_{0}\left\|D\left(u^{\varepsilon}\right)\right\|_{L^{\infty}\left([0, T] ; L^{2}(\Omega)\right)}^{2}+\frac{1}{\varepsilon}\left\|u^{\varepsilon}-\bar{u}\right\|_{L^{\infty}\left([0, T] ; L^{2}(B(\cdot))\right)}^{2} \\
& \leq C\|f\|_{L^{\infty}\left([0, T] ; L^{2}(\Omega)\right)}\|\bar{u}\|_{L^{\infty}\left([0, T] ; H^{2}(B(\cdot))\right)} \\
& +C^{\prime}\left(\|f\|_{L^{\infty}\left([0, T] ; L^{2}(\Omega)\right)}^{2}+\|\Phi\|_{L^{\infty}(\mathbb{R})}^{2}\|\bar{u}\|_{L^{\infty}\left([0, T] ; H^{2}(B(\cdot))\right)}{ }^{2}\right) . \\
& \mu_{0}\left\|D\left(u^{\varepsilon}\right)\right\|_{L^{2}\left([0, T] ; L^{2}(\Omega)\right)}^{2}+\frac{1}{\varepsilon}\left\|u^{\varepsilon}-\bar{u}\right\|_{L^{2}\left([0, T] ; L^{2}(B(\cdot))\right)}^{2} \\
& \leq C\|f\|_{L^{2}\left([0, T] ; L^{2}(\Omega)\right)}\|\bar{u}\|_{L^{\infty}\left([0, T] ; H^{2}(B(\cdot))\right)} \\
& +C^{\prime}\left(\|f\|_{L^{2}\left([0, T] ; L^{2}(\Omega)\right)}^{2}+\|\Phi\|_{\left.L^{\infty}(\mathbb{R})\right)}^{2}\|\bar{u}\|_{L^{\infty}\left([0, T] ; H^{2}(B(\cdot))\right)}{ }^{2}\right) .
\end{aligned}
$$

Definition 3.6. $(u, p, \alpha)$ is a weak solution to (3.1) with the conditions (3.2) if it fulfills

- for all $2 \leq q \leq+\infty, u \in \mathrm{L}^{q}(0, T ; V(\Omega)), p \in \mathrm{L}^{q}\left(0, T ; \mathrm{L}_{0}^{2}(\Omega \backslash \bar{B}(\cdot))\right)$ and $\alpha \in \mathrm{L}^{\infty}\left(0, T ; \mathrm{H}^{2}(\Omega)\right) \cap$ $\mathrm{L}^{2}\left(0, T ; \mathrm{H}^{3}(\Omega)\right) \cap \mathrm{H}^{1}\left(0, T ; \mathrm{H}^{1}(\Omega)\right)$,

- $u=\bar{u}$ in $O_{p}$,

- $(u, p) \in \mathrm{L}^{q}\left(0, T ; \mathrm{H}^{1}(\Omega \backslash \bar{B}(\cdot))\right) \times \mathrm{L}^{q}\left(0, T ; \mathrm{L}_{0}^{2}(\Omega \backslash \bar{B}(\cdot))\right)$, with $2 \leq q \leq+\infty$, is the solution to

$$
\left\{\begin{array}{l}
-\operatorname{div}(2 \mu D(u))=f-\nabla p \text { in } \mathrm{L}^{2}\left(0, T ; \mathrm{H}^{-1}(\Omega \backslash \bar{B}(\cdot))\right), \\
\operatorname{div} u=0 \text { in } O_{p}^{c} \\
u_{\mid \Sigma}=\bar{u}_{\mid \Sigma} \text { a.e. } \\
u \cdot \nu=\frac{\partial u_{1}}{\partial \nu}=\frac{\partial u_{2}}{\partial \nu}=0 \text { on }[0, T] \times \Gamma_{L} \\
u \text { has periodic conditions on }[0, T] \times \partial \Gamma \times[0, L],
\end{array}\right.
$$

- $\alpha$ is the solution to $(3.5)$;

- $\mu=\Phi(\alpha)$.

Theorem 3.7. Under the assumptions of Theorem 3.5 and with the extra assumption $\Phi \in W^{3, \infty}(\mathbb{R})$, there exists a unique weak solution $(u, p, \alpha)$ to (3.1)-(3.2). Moreover the weak solution $\left(u^{\varepsilon}, p^{\varepsilon}, \alpha^{\varepsilon}\right)$ of (3.3) converges weakly towards $(u, p, \alpha)$ as $\varepsilon$ goes to zero. 
We first present in Section 3.2 some Sobolev inequalities and embeddings needed in the following. We pursue in Section 3.3 with the study of the time-independent penalized Stokes problem and prove for our particular case existence, uniqueness and regularity results that we generalize in Section 3.4 to the time-dependent Stokes problem. We remind next some classical results on the convection-diffusion equation in Section 3.5. In Section 3.6 we introduce a recurring sequence and prove thanks to a fixed-point method Theorem 3.5 from which we deduce in Section 3.7 Theorem 3.7.

\subsection{Technical arguments}

Lemma 3.8. Let $m \in \mathbb{N}^{*}$. There exists a constant $C$ depending only on $\Omega$ and $m$ such that for all $v \in H^{m}(\Omega)$ we have

$$
\|v\|_{H^{m}(\Omega)} \leq C\left(\|v\|_{L^{2}(\Omega)}+\|\operatorname{div} v\|_{H^{m-1}(\Omega)}+\|\operatorname{curl} v\|_{H^{m-1}(\Omega)}+\|v \cdot \nu\|_{H^{m-1 / 2}(\partial \Omega)}\right)
$$

where $\nu$ is the outward unitary normal on $\partial \Omega$.

There exists a constant $C$ depending only on $\Omega$ and $m$ such that for all $v \in H^{m}(\Omega)$ with periodic conditions on $\partial \Gamma \times[0, L]$ we have

$$
\|v\|_{H^{m}(\Omega)} \leq C\left(\|v\|_{L^{2}(\Omega)}+\|\operatorname{div} v\|_{H^{m-1}(\Omega)}+\|\operatorname{curl} v\|_{H^{m-1}(\Omega)}+\|v \cdot \nu\|_{H^{m-1 / 2}(\partial \Gamma \times[0, L])}\right) .
$$

Proof. See [21] for the proof. The periodic version is easily deduced from [18] Section VII.6.1 and [21].

Lemma 3.9. Let $v \in H^{2}(\Omega)$ such that $v$ has periodic conditions on $\partial \Gamma \times[0, L]$ and $\frac{\partial v}{\partial \nu}=0$ on $\Gamma_{0} \cup \Gamma_{L}$. There exists then a constant $C$ depending only on $\Omega$ such that,

$$
\|v\|_{H^{2}(\Omega)} \leq C\left(\|v\|_{L^{2}(\Omega)}+\|\Delta v\|_{L^{2}(\Omega)}\right) .
$$

Moreover, if $v \in H^{3}(\Omega)$, there exists a constant $C$ depending only on $\Omega$ such that we have

$$
\|\nabla v\|_{H^{2}(\Omega)} \leq C\left(\|\nabla v\|_{L^{2}(\Omega)}+\|\Delta v\|_{L^{2}(\Omega)}+\|\nabla \Delta v\|_{L^{2}(\Omega)}\right) .
$$

Proof. The first inequality comes from the regularity of the operator $A=I-\Delta$ with the domain

$$
D(A)=\left\{u \in \mathrm{H}^{2}(\Omega), \frac{\partial u}{\partial \nu}=0 \text { on } \Gamma_{0} \cup \Gamma_{L}, u \text { has periodic conditions on } \partial \Gamma \times[0, L]\right\} .
$$

(See [3] for example). The second inequality is deduced from Lemma 3.8.

Proposition 3.10. Let $\Omega$ a regular open bounded set of $\mathbb{R}^{3}$. Let $v \in H^{1}(\Omega)$. There exists then a constant $C$ depending only on $\Omega$ such that

$$
\|v\|_{L^{3}(\Omega)} \leq C\|v\|_{L^{2}(\Omega)}^{1 / 2}\|v\|_{H^{1}(\Omega)}^{1 / 2} .
$$

Let $v \in H^{2}(\Omega)$. There exists then a constant $C$ depending only on $\Omega$ such that

$$
\|v\|_{L^{\infty}(\Omega)} \leq C\|v\|_{H^{1}(\Omega)}^{1 / 4}\|v\|_{H^{2}(\Omega)}^{3 / 4} .
$$

Proof. These two inequalities are deduced from the Sobolev embeddings of $\mathrm{H}^{1 / 2}(\Omega)$ and $\mathrm{H}^{7 / 4}(\Omega)$ respectively into $\mathrm{L}^{3}(\Omega)$ and $\mathrm{L}^{\infty}(\Omega)$ (see [2], Sect. 4) and from the estimates of the $\mathrm{H}^{1 / 2}(\Omega)$ and $\mathrm{H}^{7 / 4}(\Omega)$ norm thanks to the interpolation space theory (see [30] Sect. I).

Theorem 3.11. Let $V$ and $W$ two Hilbert spaces then

$$
E_{2,2}=\left\{v \in L^{2}(0, T ; V), \frac{\mathrm{d} v}{\mathrm{~d} t} \in L^{2}(0, T ; W)\right\}
$$

continuously maps into $\mathcal{C}^{0}\left([0, T] ;[V, W]_{\frac{1}{2}}\right)$ where $[V, W]_{\frac{1}{2}}$ is the interpolated space of order $\frac{1}{2}$ of $V$ and $W$. 
Proof. See [30] Section I.3 and [8] Section II.5.4.

Proposition 3.12 (Trace and lifting theorems in a time-dependent domain $B(t)=\Psi(t, B)$ ).

Let $B$ an open set in $\Omega$ having the uniform $\mathcal{C}^{2}$-regularity property and $\Psi \in \mathcal{C}^{0}\left([0, T] ; \mathcal{C}^{2}(\Omega)\right.$ such that for all $t \in[0, T] \Psi(t)$ is a $\mathcal{C}^{2}$-diffeomorphism on $\Omega$. There exists a time-independent constant $C>0$ such that for all $t \in[0, T]$ and for all $v \in H^{2}(B(t))$

$$
\left\|v_{\mid \partial B(t)}\right\|_{H^{3 / 2}(\partial B(t))} \leq C\|v\|_{H^{2}(B(t))} .
$$

There exists a time-independent constant $C^{\prime}>0$ such that for all $t \in[0, T]$ and for all $g \in H^{3 / 2}(\partial B(t))$ there exists a lifting $v$ of $g$ in $H^{2}(B(t))$ such that

$$
\|v\|_{H^{2}(B(t))} \leq C^{\prime}\|g\|_{H^{3 / 2}(\partial B(t))} .
$$

Proof. Thanks to the function $\Psi$ we transport the classical trace and lifting theorem in the time-dependent context. Since $\Psi \in \mathcal{C}^{0}\left([0, T] ; \mathcal{C}^{2}(\Omega)\right)$ and $\Psi(t)$ is a $\mathcal{C}^{2}$-diffeomorphism for all $t \in[0, T]$ there exists some constants $(a, b) \in\left(\mathbb{R}^{+*}\right)^{2}$ such that

$$
\forall(t, x) \in[0, T] \times \Omega \quad a \leq J(t, x) \leq b,
$$

where $J(t, x)=\left|\operatorname{det} \nabla_{x} \Psi\right|(t, x)$. Moreover there exists some constants $\left(c, c^{\prime}\right) \in\left(\mathbb{R}^{+*}\right)^{2}$ such that $\forall(t, x) \in$ $[0, T] \times \Omega$

$$
\|\Psi(t, \cdot)\|_{\mathrm{W}^{2, \infty}(\Omega)} \leq c \text { and }\left\|\Psi^{-1}(t, \cdot)\right\|_{W^{2, \infty}(\Omega)} \leq c^{\prime} .
$$

Let $v \in \mathrm{H}^{2}(B(t))$ and $\forall x \in B$ let $w_{t}(x)=v(\Psi(t, x))$. There exists some constant $K_{a, c}>0$ depending only on $(a, c)$ such that

$$
\forall t \in[0, T] w_{t} \in \mathrm{H}^{2}(B) \text { and }\left\|w_{t}\right\|_{\mathrm{H}^{2}(B)} \leq K_{a, c}\|v\|_{\mathrm{H}^{2}(B(t))} .
$$

In the same way, by writing $v(y)=w_{t}\left(\Psi^{-1}(t, y)\right)$ for all $y \in B_{t}$, there exists some constant $K_{b, c^{\prime}}^{\prime}>0$ such that

$$
\forall t \in[0, T]\|v\|_{\mathrm{H}^{2}(B(t))} \leq K_{b, c^{\prime}}^{\prime}\left\|w_{t}\right\|_{\mathrm{H}^{2}(B)} .
$$

Following [35] Section 3.8, the spaces $\mathrm{H}^{s}(\Omega)$, where $\Omega \subset \mathbb{R}^{n}$ and $s=k+\theta, k \in \mathbb{N}, 0<\theta<1$, is defined by

$$
\mathrm{H}^{s}(\Omega)=\left\{v \in \mathrm{H}^{k}(\Omega), \quad \int_{\Omega} \int_{\Omega} \frac{\left|D^{\alpha} v(x)-D^{\alpha} v(y)\right|^{2}}{|x-y|^{n+2 \theta}} \mathrm{d} x \mathrm{~d} y<+\infty \forall \alpha \in \mathbb{N}^{n} \text { such that }|\alpha|=k\right\}
$$

with the norm

$$
\|v\|_{\mathrm{H}^{s}(\Omega)}=\left(\|v\|_{\mathrm{H}^{k}(\Omega)}^{2}+\sum_{\alpha \in \mathbb{N}^{n},|\alpha|=k} \int_{\Omega} \int_{\Omega} \frac{\left|D^{\alpha} v(x)-D^{\alpha} v(y)\right|^{2}}{|x-y|^{n+2 \theta}} \mathrm{d} x \mathrm{~d} y\right)^{1 / 2} .
$$

Using this definition for the space $\mathrm{H}^{3 / 2}\left(\partial B_{t}\right)$ we obtain in the same way the existence of two constants $\left(C, C^{\prime}\right) \in$ $\left(\mathbb{R}^{+*}\right)^{2}$ such that

$$
\forall t \in[0, T] \quad C\left\|w_{t}\right\|_{\mathrm{H}^{3 / 2}(\partial B)} \leq\|v\|_{\mathrm{H}^{3 / 2}(\partial B(t))} \leq C^{\prime}\left\|w_{t}\right\|_{\mathrm{H}^{3 / 2}(\partial B)} .
$$

We now apply the classical trace and lifting theorem to the function $w_{t}$ and obtain the desired results thanks to the previous inequalities.

Remark 3.13. The lifting theorem can also be generalized in $\Omega \backslash \bar{B}(t)$ with time independent-constants to the case where we have periodic boundary conditions on $[0, L] \times \partial \Gamma, v \cdot \nu=\frac{\partial v_{1}}{\partial \nu}=\frac{\partial v_{2}}{\partial \nu}=0$ on $\Gamma_{L}$ and $v_{\mid \partial B(t)}=g$. 


\subsection{Analysis of the Stokes problem}

In the following we are interested in penalized Stokes problems where $\varepsilon$ is a constant. We then denote $u^{\varepsilon}$ by $u$. In Section 3.7 we will again distinguish $u$ and $u^{\varepsilon}$ since we study the limit as $\varepsilon$ goes to zero.

We are then interested in the following penalized Stokes problem

$$
\left\{\begin{array}{l}
-2 \operatorname{div}(\mu D(u))+\frac{\mathbf{1}_{B}}{\varepsilon}(u-\bar{u})=f-\nabla p \quad \text { in } \Omega, \\
\operatorname{div} u=0 \text { in } \Omega, \\
u=0 \text { on } \Gamma_{0} \\
u \cdot \nu=\frac{\partial u_{1}}{\partial \nu}=\frac{\partial u_{2}}{\partial \nu}=0 \text { on } \Gamma_{L}, \\
u \text { has periodic conditions on } \partial \Gamma \times[0, L],
\end{array}\right.
$$

where $\varepsilon>0, \mu \in \mathcal{F}\left(\Omega ; \mathbb{R}^{+}\right), B$ an open set in $\Omega, \bar{u} \in \mathcal{F}\left(B ; \mathbb{R}^{3}\right)$ fulfilling $\operatorname{div} \bar{u}=0, f \in \mathcal{F}\left(\Omega ; \mathbb{R}^{3}\right)$ are given, $D(u)=\frac{1}{2}\left(\nabla u+(\nabla u)^{T}\right)$ with $u \in \mathcal{F}\left(\Omega ; \mathbb{R}^{3}\right)$ and $p \in \mathcal{F}(\Omega ; \mathbb{R})$ the unknowns.

We consider at first a time-independent problem. To obtain the following results we assume (H1) and we need an additional assumption on $\bar{u}(\mathrm{H} 4)$ which is a simpler time-independent version of (H3).

\section{Hypothesis 4. (H4)}

- $\bar{u} \in H^{2}(B)$;

- $\operatorname{div} \bar{u}=0$ in $B$;

- $\bar{u}_{\mid \Gamma_{0}}=0$;

- $\bar{u}$ has periodic boundary conditions on $\partial B \cap(\partial \Gamma \times[0, L])$.

\subsubsection{Existence and uniqueness}

Definition 3.14. We associate to Stokes problem (3.6) the following variational formulation for all $v \in V(\Omega)$,

$$
\int_{\Omega} 2 \mu D(u): D(v) \mathrm{d} x+\frac{1}{\varepsilon} \int_{B}(u-\bar{u}) \cdot v \mathrm{~d} x=\int_{\Omega} f v \mathrm{~d} x .
$$

Theorem 3.15. Let $\varepsilon>0, \mu_{0}>0$ and $\mu \in L^{\infty}(\Omega)$ such that $\mu_{0} \leq \mu(x)$ a.e. in $\Omega$. We moreover assume (H1) and (H4) and $f \in L^{2}(\Omega)$. There exists a unique $(u, p) \in V(\Omega) \times L_{0}^{2}(\Omega)$ weak solution of (3.6).

Proof. This proof is inspired from various results from [8] Section II.3.2.

Thanks to Korn's and Poincaré's inequalities we have

$$
\int_{\Omega} 2 \mu|D(u)|^{2} \mathrm{~d} x+\frac{1}{\varepsilon} \int_{B}|u|^{2} \mathrm{~d} x \geq 2 \mu_{0}\|D(u)\|_{\mathrm{L}^{2}(\Omega)}^{2} \geq 2 \mu_{0}\|u\|_{\mathrm{H}^{1}(\Omega)}^{2} .
$$

We then apply Lax-Milgram's theorem to obtain the existence of $u \in V(\Omega)$ solution to the variational formulation (3.7).

This implies that $g=-\operatorname{div}(2 \mu D(u))+\frac{\mathbf{1}_{B}}{\varepsilon}(u-\bar{u})-f$ belongs to $\mathrm{H}^{-1}(\Omega)$. Testing this function against a function $\phi \in \mathrm{H}_{0}^{1}(\Omega)$ such that $\operatorname{div} \phi=0$ we obtain:

$$
\langle g, \phi\rangle_{\mathrm{H}^{-1}(\Omega), \mathrm{H}_{0}^{1}(\Omega)}=\int_{\Omega} 2 \mu D(u): D(\phi) \mathrm{d} x+\frac{1}{\varepsilon} \int_{B}(u-\bar{u}) \phi \mathrm{d} x-\int_{\Omega} f \phi \mathrm{d} x=0 .
$$

De Rahm's theorem induces the existence of a function $p \in \mathrm{L}_{0}^{2}(\Omega)$ such that $g=-\nabla p$. We now verify that the last boundary conditions are fulfilled. The function $g+\nabla p$ rewrites

$$
g+\nabla p=-\operatorname{div}(2 \mu D(u)-p I)+\frac{\mathbf{1}_{B}}{\varepsilon}(u-\bar{u})-f=0 .
$$


From this expression we deduce that $2 \mu D(u)-p I$ lies in $\mathrm{H}(\operatorname{div})=\left\{v \in \mathrm{L}^{2}(\Omega)\right.$, $\left.\operatorname{div} v \in \mathrm{L}^{2}(\Omega)\right\}$ and admits a normal trace in $\mathrm{H}^{-1 / 2}(\partial \Omega)$. For all $\phi \in V(\Omega)$,

$$
\begin{aligned}
& \langle g+\nabla p, \phi\rangle_{\mathrm{H}^{-1}(\Omega), \mathrm{H}_{0}^{1}(\Omega)}=0 \\
= & \int_{\Omega}(2 \mu D(u): D(\phi)-p \operatorname{div} \phi) \mathrm{d} x-\int_{\partial \Omega}(2 \mu D(u) \cdot \nu \phi-p \nu \cdot \phi) \mathrm{d} \sigma+\frac{1}{\varepsilon} \int_{B}(u-\bar{u}) \phi \mathrm{d} x-\int_{\Omega} f \phi \mathrm{d} x .
\end{aligned}
$$

Since $\operatorname{div} \phi=0$ and thanks to (3.7) expressed with $v=\phi$ and to the boundary conditions already fulfilled by $u$ and $\phi$ we obtain

$$
\begin{aligned}
\int_{\Gamma_{L}}(2 \mu D(u)-p I) \nu \cdot \phi \mathrm{d} \sigma & =\langle(2 \mu D(u)-p I) \cdot \nu, \phi\rangle_{\mathrm{H}^{-1 / 2}\left(\Gamma_{L}\right), \mathrm{H}^{1 / 2}\left(\Gamma_{L}\right)} \\
& =\langle\mathcal{P}(2 \mu D(u) \cdot \nu), \phi\rangle_{\mathrm{H}^{-1 / 2}\left(\Gamma_{L}\right), \mathrm{H}^{1 / 2}\left(\Gamma_{L}\right)}=0
\end{aligned}
$$

for all $\phi \in V(\Omega)$ where $\mathcal{P}$ is the orthogonal projection on $\nu^{\perp}=e_{3}^{\perp}$.

Let $\psi \in\left(\mathrm{H}^{1 / 2}\left(\Gamma_{L}\right)\right)^{2}$. There exists a lifting $\phi \in V(\Omega)$ such that $\phi=(\psi, 0)$ on $\Gamma_{L}$ (see [8] Sect. III.4 for example). Then for all $\psi \in\left(\mathrm{H}^{1 / 2}\left(\Gamma_{L}\right)\right)^{2}$, we obtain thanks to its lifting in $V(\Omega)$ that

$$
\langle 2 \mu D(u) \cdot \nu, \phi\rangle_{\left(\mathrm{H}^{-1 / 2}\left(\Gamma_{L}\right)\right)^{3},\left(\mathrm{H}^{1 / 2}\left(\Gamma_{L}\right)\right)^{3}}=\langle\mathcal{Q}(2 \mu D(u) \cdot \nu), \psi\rangle_{\left(\mathrm{H}^{-1 / 2}\left(\Gamma_{L}\right)\right) 2,\left(\mathrm{H}^{1 / 2}\left(\Gamma_{L}\right)\right)^{2}}=0,
$$

where $\mathcal{Q}: \mathbb{R}^{3} \rightarrow \nu^{\perp} \cong \mathbb{R}^{2}$ is the orthogonal projection on $\nu^{\perp}$. and then that

$$
\mathcal{Q}(2 \mu D(u) . \nu)=0 \text { in }\left(\mathrm{H}^{-1 / 2}\left(\Gamma_{L}\right)\right)^{2} .
$$

This writes

$$
\mu\left(\partial_{1} u_{3}+\partial_{3} u_{1}\right)=\mu\left(\partial_{2} u_{3}+\partial_{3} u_{2}\right)=0 \text { in } \mathrm{H}^{-1 / 2}\left(\Gamma_{L}\right) .
$$

Since $\mu \geq \mu_{0}>0, u \cdot \nu \in \mathrm{H}^{1 / 2}\left(\Gamma_{L}\right)$ and $u \cdot \nu=u_{3}=0$ on $\Gamma_{L}$ we have $\partial_{1} u_{3}=\partial_{2} u_{3}=0$ and

$$
\partial_{3} u_{1}=\partial_{3} u_{2}=0 \text { on } \Gamma_{L} \text {. }
$$

Hence $(u, p) \in V(\Omega) \times \mathrm{L}_{0}^{2}(\Omega)$ is the solution to the Stokes problem (3.6).

\subsection{2. "Harmonic" prolongation of $\bar{u}$}

To obtain precise estimates on $(u, p)$ we need a prolongation $\tilde{u}$ of $\bar{u}$ in the whole domain $\Omega$. We study the following equation:

$$
\left\{\begin{array}{l}
-\operatorname{div}(2 \mu D(\tilde{u}))=-\nabla \tilde{p} \quad \text { in } \Omega \backslash \bar{B}, \\
\operatorname{div} \tilde{u}=0 \text { in } \Omega \backslash \bar{B}, \\
\tilde{u} \cdot \nu=\frac{\partial \tilde{u}_{1}}{\partial \nu}=\frac{\partial \tilde{u}_{2}}{\partial \nu}=0 \text { on } \Gamma_{L}, \\
\tilde{u} \text { has periodic conditions on } \partial \Gamma \times[l, L], \\
\tilde{u}=\bar{u} \text { on } \sigma=\partial B \backslash \partial \Omega .
\end{array}\right.
$$

Proposition 3.16. We assume (H1)-(H4) and we let $\mu_{0}>0$ and $\mu \in L^{\infty}(\Omega \backslash \bar{B})$ such that $\mu_{0} \leq \mu(x)$ a.e. in $\Omega \backslash \bar{B}$. There exists a unique weak solution $(\tilde{u}, \tilde{p})$ of $(3.8)$ with $\tilde{u} \in H^{1}(\Omega \backslash \bar{B})$ and $\tilde{p} \in L_{0}^{2}(\Omega \backslash \bar{B})$.

Moreover there exists a constant $C$ depending on $\Omega \backslash \bar{B}$ such that

$$
\|\tilde{u}\|_{H^{1}(\Omega \backslash \bar{B})}+\|\tilde{p}\|_{L_{0}^{2}(\Omega \backslash \bar{B})} \leq C\|\bar{u}\|_{H^{2}(B)} .
$$

Proof. Since $\bar{u} \in \mathrm{H}^{2}(B)$ and $B$ fulfills $(\mathrm{H} 1), \bar{u}_{\mid \sigma} \in \mathrm{H}^{3 / 2}(\sigma)$ and there exists a lifting $\phi \in \mathrm{H}^{2}(\Omega \backslash \bar{B})$ such that $\phi_{\mid \sigma}=\bar{u}$ on $\sigma, \phi \cdot \nu=\phi_{3}=\frac{\partial \phi_{1}}{\partial \nu}=\frac{\partial \phi_{2}}{\partial \nu}=0$ on $\Gamma_{L}$ and $\phi$ has periodic conditions on $\partial \Gamma \times[0, L]$. We let $\tilde{u}=w+\phi$ and find $w$, solution to the following Stokes problem:

$$
\left\{\begin{array}{l}
-2 \operatorname{div}(\mu D(w)=-\nabla \tilde{p}+2 \operatorname{div}(\mu D(\phi)) \quad \text { in } \Omega \backslash \bar{B}, \\
\operatorname{div} w=-\operatorname{div} \phi \text { in } \Omega \backslash \bar{B}, \\
\tilde{w} \cdot \nu=\frac{\partial w_{1}}{\partial \nu}=\frac{\partial w_{2}}{\partial \nu}=0 \text { on } \Gamma_{L}, \\
w \text { has periodic conditions on } \partial \Gamma \times[l, L], \\
w=0 \text { on } \sigma .
\end{array}\right.
$$


The existence and the estimates are classical (cf. [8] Sect. III.4 and the proof of Thm. 3.15).

Proposition 3.17. Under the assumptions of Proposition 3.16, we let $P(\bar{u})=\mathbf{1}_{B} \bar{u}+\mathbf{1}_{\Omega \backslash \bar{B}} \tilde{u}$. Then $P(\bar{u}) \in V(\Omega)$ and there exists a constant $C$ depending on $B$ (and $\Omega$ ) such that

$$
\|P(\bar{u})\|_{H^{1}(\Omega)} \leq C\|\bar{u}\|_{H^{2}(B)} .
$$

\subsubsection{Estimates on $(u, p)$}

Under the assumptions of Theorems 3.15 and Proposition 3.16 we let $(u, p)$ the unique weak solution to (3.6) given by Theorem 3.15. Applying (3.7) with $v=u-P(\bar{u})$, we obtain

$$
\begin{aligned}
2 \mu_{0}\|D(u)\|_{\mathrm{L}^{2}(\Omega)}^{2}+\frac{1}{\varepsilon}\|u-\bar{u}\|_{\mathrm{L}^{2}(B)}^{2} \leq & \|f\|_{\mathrm{L}^{2}(\Omega)}\left(\|u\|_{\mathrm{L}^{2}(\Omega)}+\|P(\bar{u})\|_{\mathrm{L}^{2}(\Omega)}\right) \\
& +2\|\mu\|_{\mathrm{L}^{\infty}(\Omega)}\|D(P(\bar{u}))\|_{\mathrm{L}^{2}(\Omega)}\|D(u)\|_{\mathrm{L}^{2}(\Omega)},
\end{aligned}
$$

Hence

$$
\begin{aligned}
\mu_{0}\|D(u)\|_{\mathrm{L}^{2}(\Omega)}^{2}+\frac{1}{\varepsilon}\|u-\bar{u}\|_{\mathrm{L}^{2}(B)}^{2} \leq & C\|f\|_{\mathrm{L}^{2}(\Omega)}\|\bar{u}\|_{\mathrm{H}^{2}(B)} \\
& +C^{\prime}\left(\|f\|_{\mathrm{L}^{2}(\Omega)}^{2}+\|\mu\|_{\mathrm{L}^{\infty}(\Omega)}^{2}\|\bar{u}\|_{\mathrm{H}^{2}(B)}^{2}\right) .
\end{aligned}
$$

From (3.6), we deduce

$$
\begin{aligned}
\|\nabla p\|_{\mathrm{H}^{-1}(\Omega)} & \leq\|f\|_{\mathrm{H}^{-1}(\Omega)}+\|\operatorname{div}(2 \mu D(u))\|_{\mathrm{H}^{-1}(\Omega)}+\frac{1}{\varepsilon}\left\|\mathbf{1}_{B}(u-\bar{u})\right\|_{\mathrm{H}^{-1}(\Omega)} \\
& \left.\leq\|f\|_{\mathrm{L}^{2}(\Omega)}+\|\mu\|_{\mathrm{L}^{\infty}(\Omega)} \| D(u)\right)\left\|_{\mathrm{L}^{2}(\Omega)}+\frac{1}{\varepsilon}\right\| u-\bar{u} \|_{\mathrm{L}^{2}(B)} .
\end{aligned}
$$

Since the mean of $p$ is null, Poincaré's lemma gives

$$
\left.\|p\|_{L_{0}^{2}(\Omega)} \leq C\left(\|f\|_{L^{2}(\Omega)}+\|\mu\|_{L^{\infty}(\Omega)} \| D(u)\right)\left\|_{L^{2}(\Omega)}+\frac{1}{\varepsilon}\right\| u-\bar{u} \|_{L^{2}(B)}\right) .
$$

\subsubsection{Regularity of the solution}

Theorem 3.18. Under the assumptions of Theorem 3.15 and with the additional assumption $\mu \in W^{1, \infty}(\Omega)$, the unique solution $(u, p)$ of $(3.6)$ belongs to $H^{2}(\Omega) \times H^{1}(\Omega)$ and there exists some constant $C=C(\Omega)$ such that

$$
\|u\|_{H^{2}(\Omega)}+\left\|\frac{p}{\mu}\right\|_{H^{1}(\Omega)} \leq C\|g\|_{L^{2}(\Omega)},
$$

where $g=\frac{1}{\mu}\left(2 D(u) \nabla \mu+f-\frac{\mathbf{1}_{B}}{\varepsilon}(u-\bar{u})+p \frac{\nabla \mu}{\mu}\right)$.

Proof. The weak solution $u$ to (3.6) is also solution to

$$
\left\{\begin{array}{l}
-2 \operatorname{div}(D(u))=g-\nabla p^{\prime} \quad \text { in } \Omega, \\
\operatorname{div} u=0 \text { in } \Omega, \\
u=0 \text { on } \Gamma_{0} \\
u \cdot \nu=\frac{\partial u_{1}}{\partial \nu}=\frac{\partial u_{2}}{\partial \nu}=0 \text { on } \Gamma_{L}, \\
u \text { has periodic conditions on } \partial \Gamma \times[0, L],
\end{array}\right.
$$

where $g=\frac{1}{\mu}\left(2 D(u) \nabla \mu+f-\frac{1_{B}}{\varepsilon}(u-\bar{u})+p \frac{\nabla \mu}{\mu}\right) \in \mathrm{L}^{2}(\Omega)$ and $p^{\prime}=\frac{p}{\mu}$.

Following the classical regularity results for the Stokes equation (see [8] Sect. III.4), we obtain $\left(u, p^{\prime}\right) \in$ $\mathrm{H}^{2}(\Omega) \times \mathrm{H}^{1}(\Omega)$ and then $(u, p) \in \mathrm{H}^{2}(\Omega) \times \mathrm{H}^{1}(\Omega)$ and the announced inequalities. 


\subsection{Stokes problem with time dependent domain}

Let $T>0$. In the following the domain $B$ depends on the time $t \in[0, T]$ and we need to track the dependency in the time $t$ of the constants (especially the ones in all Sobolev related theorems). Using classical trace and lifting theorem (on the domain $B(t)$ or $\Omega \backslash \bar{B}(t)$ ) there appears some constants depending on $B(t)$ and $\Omega \backslash \bar{B}(t)$. Thanks to Proposition 3.12 we can estimate the time-behaviour of these constants.

Proposition 3.19 (Time dependent "harmonic" lifting). Let us assume (H1)-(H2)-(H3), $\mu_{0}>0$ and $\mu \in$ $L^{\infty}\left(O_{p}^{c}\right)$ such that $\mu_{0} \leq \mu(t, x)$ a.e. in $O_{p}^{c}$, there exists a unique weak solution $(\tilde{u}, \tilde{p})$ to

$$
\left\{\begin{array}{l}
-2 \operatorname{div}(\mu D(\tilde{u}))=-\nabla \tilde{p} \quad \text { in } O_{p}^{c}, \\
\operatorname{div} \tilde{u}=0 \text { in } O_{p}^{c}, \\
\tilde{u} \cdot \nu=\frac{\partial \tilde{u}_{1}}{\partial \nu}=\frac{\partial \tilde{u}_{2}}{\partial \nu}=0 \quad \text { on }[0, T] \times \Gamma_{L}, \\
\tilde{u} \text { has periodic conditions on }[0, T] \times \partial \Gamma \times[l, L], \\
\tilde{u}=\bar{u} \text { a.e. on } \Sigma,
\end{array}\right.
$$

where $\tilde{u} \in L^{\infty}\left(0, T ; H^{1}(\Omega \backslash \bar{B}(\cdot))\right.$ and $\tilde{p} \in L^{\infty}\left(0, T ; L_{0}^{2}(\Omega \backslash \bar{B}(\cdot))\right)$.

Moreover there exists a constant $C$ depending on $B$ and $\Psi$ such that for all $1 \leq q \leq+\infty$

$$
\|\tilde{u}\|_{L^{q}\left(0, T ; H^{1}(\Omega \backslash \bar{B}(\cdot))\right)}+\|\tilde{p}\|_{L^{q}\left(0, T ; L_{0}^{2}(\Omega \backslash \bar{B}(\cdot))\right)} \leq C\|\bar{u}\|_{H^{3}\left(O_{p}\right)} .
$$

Proof. Since $\bar{u} \in \mathcal{C}^{0}\left(0, T ; \mathrm{H}^{2}(B(\cdot))\right)$ and the domain $B(t)$ fulfills (H1) and (H2), we have thanks to Proposition 3.12 and the remark that follows that $\bar{u}_{\mid \Sigma} \in \mathrm{L}^{\infty}\left([0, T] ; \mathrm{H}^{3 / 2}(\partial B(\cdot))\right)$ and there exists a lifting $\phi \in$ $\mathrm{L}^{\infty}\left(0, T ; \mathrm{H}^{2}(\Omega \backslash \bar{B}(\cdot))\right)$ such that $\phi_{\mid \Sigma}=\bar{u}$ on $\Sigma$, and for all $t \in[0, T], \phi \cdot \nu=\phi_{3}=\frac{\partial \phi_{1}}{\partial \nu}=\frac{\partial \phi_{2}}{\partial \nu}=0$ on $\Gamma_{L}$ and $\phi$ has periodic conditions on $\partial \Gamma \times[l, L]$ and there exists some constants $C_{1}$ and $C_{2}$ depending only on $B$ and $\Psi$ such that

$$
\|\phi\|_{\mathrm{L}^{\infty}\left(0, T ; \mathrm{H}^{2}(\Omega \backslash \bar{B}(\cdot))\right)} \leq C_{1}\|\bar{u}\|_{\mathrm{L}^{\infty}\left([0, T] ; \mathrm{H}^{3 / 2}(\partial B(t) \backslash \partial \Omega)\right.} \leq C_{2}\|\bar{u}\|_{\mathrm{L}^{\infty}\left(0, T ; \mathrm{H}^{2}(B(\cdot))\right)},
$$

and $\phi \in \mathrm{L}^{\infty}\left(0, T ; \mathrm{H}^{2}(\Omega \backslash \bar{B}(\cdot))\right)$. As in the proof of Proposition 3.16 we obtain for all $t \in[0, T]$ the existence of a unique solution $\tilde{u}$ to $(3.10)$ that fulfills

$$
\|\tilde{u}(t, \cdot)\|_{\mathrm{H}^{1}(\Omega \backslash \bar{B}(t))}+\|\tilde{p}(t, \cdot)\|_{\mathrm{L}^{2}(\Omega \backslash \bar{B}(t))} \leq C\|\phi\|_{\mathrm{L}^{\infty}\left(0, T ; \mathrm{H}^{2}(\Omega \backslash \bar{B}(\cdot))\right)} \leq C^{\prime}\|\bar{u}\|_{\mathrm{L}^{\infty}\left([0, T] ; \mathrm{H}^{2}(B(\cdot))\right)} \quad \forall t \in[0, T],
$$

hence the result.

Proposition 3.20. Under the assumptions of Proposition 3.19 we let $P(\bar{u})=\mathbf{1}_{O_{p}} \bar{u}+\mathbf{1}_{O_{p}^{c}} \tilde{u}$. Then $P(\bar{u}) \in$ $L^{\infty}(0, T ; V(\Omega))$ and there exists a constant $C$ depending on $B$ and $\Psi$ such that for all $1 \leq q \leq+\infty$,

$$
\|P(\bar{u})\|_{L^{q}\left(0, T ; H^{1}(\Omega)\right)} \leq C\|\bar{u}\|_{L^{\infty}\left([0, T] ; H^{2}(B(\cdot))\right)} .
$$

Following the scheme we use in Section 3.3 we obtain the following theorem.

Theorem 3.21. We assume (H1), (H2) and (H3). Let $\mu_{0}>0, \varepsilon>0$ and $T>0$. Let $\mu \in L^{\infty}([0, T] \times \Omega)$ such that $\mu_{0} \leq \mu(t, x)$ a.e. in $[0, T] \times \Omega$. Let $f \in L^{\infty}\left(0, T ; L^{2}(\Omega)\right)$. There exists then a unique weak solution $(u, p)$ to the problem

$$
\left\{\begin{array}{l}
-\operatorname{div}(2 \mu D(u))+\frac{\boldsymbol{1}_{B(t)}}{\varepsilon}(u-\bar{u})=f-\nabla p \quad \text { in }[0, T] \times \Omega, \\
\operatorname{div} u=0 \text { in }[0, T] \times \Omega, \\
u=0 \text { on }[0, T] \times \Gamma_{0}, \\
u \cdot \nu=\frac{\partial u_{1}}{\partial \nu}=\frac{\partial u_{2}}{\partial \nu}=0 \quad \text { on }[0, T] \times \Gamma_{L}, \\
u \text { has periodic conditions on }[0, T] \times \partial \Gamma \times[0, L],
\end{array}\right.
$$


which fulfills

$$
(u, p) \in L^{q}([0, T], V(\Omega)) \times L^{q}\left([0, T] ; L_{0}^{2}(\Omega)\right)
$$

for all $2 \leq q \leq+\infty$. We also have the following inequalities:

$$
\begin{aligned}
& \mu_{0}\|D(u)\|_{L^{\infty}\left([0, T] ; L^{2}(\Omega)\right)}^{2}+\frac{1}{\varepsilon}\|u-\bar{u}\|_{L^{\infty}\left([0, T] ; L^{2}(B(\cdot))\right)}^{2} \\
& \leq C\|f\|_{L^{\infty}\left([0, T] ; L^{2}(\Omega)\right)}\|\bar{u}\|_{L^{\infty}\left([0, T] ; H^{2}(B(\cdot))\right)} \\
& +C^{\prime}\left(\|f\|_{L^{\infty}\left([0, T] ; L^{2}(\Omega)\right)}^{2}+\|\mu\|_{L^{\infty}([0, T] \times \Omega)}^{2}\|\bar{u}\|_{L^{\infty}\left([0, T] ; H^{2}(B(\cdot))\right)}^{2}\right) . \\
& \mu_{0}\|D(u)\|_{L^{2}\left([0, T] ; L^{2}(\Omega)\right)}^{2}+\frac{1}{\varepsilon}\|u-\bar{u}\|_{L^{2}\left([0, T] ; L^{2}(B(\cdot))\right)}^{2} \\
& \leq C\|f\|_{L^{2}\left([0, T] ; L^{2}(\Omega)\right)}\|\bar{u}\|_{L^{\infty}\left([0, T] ; H^{2}(B(\cdot))\right)} \\
& +C^{\prime}\left(\|f\|_{L^{2}\left([0, T] ; L^{2}(\Omega)\right)}^{2}+\|\mu\|_{L^{2}\left([0, T] ; L^{\infty}(\Omega)\right)}^{2}\|\bar{u}\|_{L^{\infty}\left([0, T] ; H^{2}(B(\cdot))\right)}^{2}\right) .
\end{aligned}
$$

Moreover, if $\mu \in L^{q}\left(0, T ; W^{1, \infty}(\Omega)\right)$, with $2 \leq q \leq+\infty$,

$$
(u, p) \in L^{q}\left([0, T], H^{2}(\Omega)\right) \times L^{q}\left([0, T] ; H^{1}(\Omega)\right) .
$$

and there exists a constant $C$ depending on $\Omega$ such that

$$
\|u\|_{L^{q}\left([0, T] ; H^{2}(\Omega)\right)}+\left\|\frac{p}{\mu}\right\|_{L^{q}\left([0, T] ; H^{1}(\Omega)\right)} \leq C\|g\|_{L^{q}\left([0, T] ; L^{2}(\Omega)\right)},
$$

where $g=\frac{1}{\mu}\left(2 D(u) \nabla \mu+f-\frac{\mathbf{1}_{B(t)}}{\varepsilon}(u-\bar{u})+p \frac{\nabla \mu}{\mu}\right) \in L^{q}\left([0, T] ; L^{2}(\Omega)\right)$.

\subsection{Convection-diffusion equation}

Theorem 3.22. Let $\eta>0$ and $u \in L^{q}(0, T ; V(\Omega))$ for all $2 \leq q \leq+\infty$ such that $u \cdot \nu=\frac{\partial u_{1}}{\partial \nu}=\frac{\partial u_{2}}{\partial \nu}=0$ on $\Gamma_{L}$. Let $\alpha_{0} \in H^{1}(\Omega)$. There exists a unique solution $\alpha \in L^{\infty}\left(0, T ; H^{1}(\Omega)\right) \cap L^{2}\left(0, T ; H^{2}(\Omega)\right) \cap H^{1}\left(0, T ; L^{2}(\Omega)\right)$ to the problem

$$
\left\{\begin{array}{l}
\partial_{t} \alpha+u \cdot \nabla \alpha-\eta \Delta \alpha=0 \quad \text { in } \Omega, \\
\frac{\partial \alpha}{\partial \nu}=0 \quad \text { on } \Gamma_{0} \cup \Gamma_{L}, \\
\alpha \text { has periodic conditions on } \partial \Gamma \times[0, L], \\
\alpha(0, \cdot)=\alpha_{0} \text { in } \Omega .
\end{array}\right.
$$

Moreover if $\alpha_{0} \in H^{2}(\Omega)$ then $\alpha \in L^{\infty}\left(0, T ; H^{2}(\Omega)\right) \cap L^{2}\left(0, T ; H^{3}(\Omega)\right) \cap H^{1}\left(0, T ; H^{1}(\Omega)\right)$ and there exists some constants $C$ and $C^{\prime}$ depending only on $\Omega$ and $\eta$ such that for all $t \in[0, T]$ :

$$
\begin{aligned}
& \|\alpha(t, \cdot)\|_{H^{2}(\Omega)} \leq C\left\|\alpha_{0}\right\|_{H^{2}(\Omega)} \exp \left(C^{\prime} \int_{0}^{t}\left(1+\|u(s, \cdot)\|_{H^{1}(\Omega)}^{2}+\|u(s, \cdot)\|_{H^{1}(\Omega)}^{4}+\|u(s, \cdot)\|_{H^{1}(\Omega)}^{8}\right) \mathrm{d} s\right), \\
& \|\alpha\|_{L^{2}\left(0, t ; H^{3}(\Omega)\right)} \leq C\left\|\alpha_{0}\right\|_{H^{2}(\Omega)} \exp \left(C^{\prime} \int_{0}^{t}\left(1+\|u(s, \cdot)\|_{H^{1}(\Omega)}^{2}+\|u(s, \cdot)\|_{H^{1}(\Omega)}^{4}+\|u(s, \cdot)\|_{H^{1}(\Omega)}^{8}\right) \mathrm{d} s\right) .
\end{aligned}
$$

Proof. We perform a classical Galerkin decomposition and energy estimates (see also [17] vol. 5, Sect. XVIII). Using Lemma 3.9 and Proposition 3.10 we obtain

$$
Y(t)+\eta \int_{0}^{t} Z(s) \mathrm{d} s \leq Y(0)+\int_{0}^{t} C_{\eta, \Omega} Y(s)\left(1+\|u(s, \cdot)\|_{\mathrm{H}^{1}(\Omega)}^{2}+\|u(s, \cdot)\|_{\mathrm{H}^{1}(\Omega)}^{4}+\|u(s, \cdot)\|_{\mathrm{H}^{1}(\Omega)}^{8}\right) \mathrm{d} s
$$


where $Y(t)=\|\alpha(t, \cdot)\|_{\mathrm{L}^{2}(\Omega)}^{2}+\|\Delta \alpha\|_{\mathrm{L}^{2}(\Omega)}^{2}$ and $Z(t)=\|\alpha(t, \cdot)\|_{\mathrm{L}^{2}(\Omega)}^{2}+\|\nabla \alpha(t, \cdot)\|_{\mathrm{L}^{2}(\Omega)}^{2}+\|\Delta \alpha(t, \cdot)\|_{\mathrm{L}^{2}(\Omega)}^{2}+$ $\|\nabla \Delta \alpha(t, \cdot)\|_{\mathrm{L}^{2}(\Omega)}^{2}$. Gronwall lemma then gives

$$
Y(t)+\eta \int_{0}^{t} Z(s) \mathrm{d} s \leq Y(0) \exp \int_{0}^{t}\left(1+\|u(s, \cdot)\|_{\mathrm{H}^{1}(\Omega)}^{2}+\|u(s, \cdot)\|_{\mathrm{H}^{1}(\Omega)}^{4}+\|u(s, \cdot)\|_{\mathrm{H}^{1}(\Omega)}^{8}\right) \mathrm{d} s
$$

The results are easily deduce thanks to Lemma 3.9 .

Remark 3.23. Since we use Lemma 3.9 the first inequality in Theorem 3.22 is not sharp. The second one is a very rough bound.

\subsection{Study of the penalized problem}

To prove the existence of a solution to this coupled non-linear problem, we introduce the sequence $\left(\alpha^{n}, u^{n}, p^{n}\right)$ defined by

(1) $\alpha^{0}=\alpha_{0}$;

(2) $\mu^{n}=\Phi\left(\alpha^{n}\right)$;

(3) $\left(u^{n}, p^{n}\right)$ is the solution to

$$
\left\{\begin{array}{l}
-\operatorname{div}\left(2 \mu^{n} D\left(u^{n}\right)\right)+\frac{\mathbf{1}_{B}(t)}{\varepsilon}\left(u^{n}-\bar{u}\right)=f-\nabla p^{n} \quad \text { in } \Omega, \\
\operatorname{div} u^{n}=0 \text { in } \Omega \\
u^{n}=0 \text { on } \Gamma_{0} \\
u \cdot \nu=\frac{\partial u_{1}}{\partial \nu}=\frac{\partial u_{2}}{\partial \nu}=0 \text { on } \Gamma_{L}, \\
u^{n} \text { has periodic conditions on } \partial \Gamma \times[0, L],
\end{array}\right.
$$

(4) $\alpha^{n+1}$ is the solution to

$$
\left\{\begin{array}{l}
\partial_{t} \alpha^{n+1}+u^{n} \cdot \nabla \alpha^{n+1}-\eta \Delta \alpha^{n+1}=0 \quad \text { in } \Omega, \\
\frac{\partial \alpha^{n+1}}{\partial \nu}=0 \text { on } \Gamma_{0} \cup \Gamma_{L}, \\
\alpha^{n+1} \text { has periodic conditions on } \partial \Gamma \times[0, L] \\
\alpha^{n+1}(0, \cdot)=\alpha_{0} \quad \text { in } \Omega
\end{array}\right.
$$

We let $\alpha^{n+1}=G\left(\alpha^{n}\right)$

In the following we prove that $G$ is a contraction in a well-chosen space.

\subsubsection{Study of $G$}

Let $E=\mathrm{L}^{\infty}\left(0, T ; \mathrm{H}^{1}(\Omega)\right) \cap L^{2}\left(0, T ; \mathrm{H}^{2}(\Omega)\right)$ and $F=\mathrm{L}^{\infty}\left(0, T ; \mathrm{H}^{2}(\Omega)\right) \cap L^{2}\left(0, T ; \mathrm{H}^{3}(\Omega)\right)$.

The operator $G$ maps $E$ to $E$ and $F$ to $F$ thanks to Theorems 3.21 and 3.22. Let $\left(\alpha, \alpha^{\prime}\right) \in F^{2}$ that have periodic boundary conditions on $\partial \Gamma \times[0, L]$ and homogeneous Neumann boundary conditions on $\Gamma_{0} \cup \Gamma_{L}$.

Let $\mu=\Phi(\alpha)$ and $\mu^{\prime}=\Phi\left(\alpha^{\prime}\right) .\left(\mu, \mu^{\prime}\right) \in\left(\mathrm{L}^{\infty}\left(0, T ; \mathrm{H}^{2}(\Omega)\right) \cap \mathrm{L}^{2}\left(0, T ; \mathrm{H}^{3}(\Omega)\right)\right)^{2}$.

Proposition 3.24. There exists some constant $C$ depending on $\Omega$ and $\|\Phi\|_{W^{2, \infty}(\mathbb{R})}$ such that for all $t \in[0, T]$,

$$
\left\|\left(\mu-\mu^{\prime}\right)(t, \cdot)\right\|_{H^{1}(\Omega)} \leq C\left(1+\left\|\alpha^{\prime}\right\|_{L^{\infty}\left(0, T ; H^{2}(\Omega)\right)}\right)\left\|\left(\alpha-\alpha^{\prime}\right)(t, \cdot)\right\|_{H^{1}(\Omega)} .
$$

There exists some constant $C$ depending on $\Omega$ and $\|\Phi\|_{W^{3, \infty}(\mathbb{R})}$ such that for all $t \in[0, T]$,

$$
\left\|\left(\mu-\mu^{\prime}\right)(t, \cdot)\right\|_{H^{2}(\Omega)} \leq C\left(1+\|\alpha\|_{L^{\infty}\left(0, T ; H^{2}(\Omega)\right)}\right)\left(1+\left\|\alpha^{\prime}\right\|_{L^{\infty}\left(0, T ; H^{2}(\Omega)\right)}\right)\left\|\left(\alpha-\alpha^{\prime}\right)(t, \cdot)\right\|_{H^{2}(\Omega)} .
$$


Proof.

$$
\begin{aligned}
\| \mu(t, \cdot)-\mu^{\prime}(t, \cdot) & \left\|_{\mathrm{L}^{2}(\Omega)} \leq\right\| \nabla \Phi\left\|_{\mathrm{L}^{\infty}(\mathbb{R})}\right\| \alpha(t, \cdot)-\alpha^{\prime}(t, \cdot) \|_{\mathrm{L}^{2}(\Omega)} \\
\left\|\nabla \mu(t, \cdot)-\nabla \mu^{\prime}(t, \cdot)\right\|_{L^{2}(\Omega)}= & \left\|\Phi^{\prime}(\alpha) \nabla \alpha-\Phi^{\prime}\left(\alpha^{\prime}\right) \nabla \alpha^{\prime}\right\|_{\mathrm{L}^{2}(\Omega)} \\
\leq & \left\|\Phi^{\prime}(\alpha) \nabla\left(\alpha-\alpha^{\prime}\right)\right\|_{\mathrm{L}^{2}(\Omega)}+\left\|\left(\Phi^{\prime}(\alpha)-\Phi^{\prime}\left(\alpha^{\prime}\right)\right) \nabla \alpha^{\prime}\right\|_{\mathrm{L}^{2}(\Omega)} \\
\leq & \|\Phi\|_{\mathrm{W}^{1, \infty}(\mathbb{R})}\left\|\nabla\left(\alpha-\alpha^{\prime}\right)(t, \cdot)\right\|_{\mathrm{L}^{2}(\Omega)} \\
\leq & +\|\Phi\|_{\mathrm{W}^{2, \infty}(\mathbb{R})}\left\|\alpha(t, \cdot)-\alpha^{\prime}(t, \cdot)\right\|_{\mathrm{L}^{6}(\Omega)}\left\|\nabla \alpha^{\prime}(t, \cdot)\right\|_{\mathrm{L}^{3}(\Omega)} \\
\leq & \|\Phi\|_{\mathrm{W}^{1, \infty}(\mathbb{R})}\left\|\nabla\left(\alpha-\alpha^{\prime}\right)(t, \cdot)\right\|_{\mathrm{L}^{2}(\Omega)} \\
& +C\|\Phi\|_{\mathrm{W}^{2, \infty}(\mathbb{R})}\left\|\alpha(t, \cdot)-\alpha^{\prime}(t, \cdot)\right\|_{\mathrm{H}^{1}(\Omega)}\left\|\alpha^{\prime}(t, \cdot)\right\|_{\mathrm{H}^{2}(\Omega)} .
\end{aligned}
$$

Since $\alpha^{\prime} \in F$, there exists a constant $C>0$ depending only on $\Omega$ and $\|\Phi\|_{\mathrm{W}^{2, \infty}(\mathbb{R})}$ such that

$$
\left\|\left(\mu-\mu^{\prime}\right)(t, \cdot)\right\|_{\mathrm{H}^{1}(\Omega)} \leq C\left(1+\left\|\alpha^{\prime}\right\|_{\mathrm{L}^{\infty}\left(0, T ; \mathrm{H}^{2}(\Omega)\right)}\right)\left\|\left(\alpha-\alpha^{\prime}\right)(t, \cdot)\right\|_{\mathrm{H}^{1}(\Omega)} .
$$

In the same way,

$$
\begin{aligned}
\left\|\nabla^{2}\left(\mu-\mu^{\prime}\right)\right\|_{\mathrm{L}^{2}(\Omega)}= & \left\|\Phi^{\prime \prime}(\alpha) \nabla \alpha(\nabla \alpha)^{t}+\Phi^{\prime}(\alpha) \nabla^{2} \alpha-\Phi^{\prime \prime}\left(\alpha^{\prime}\right) \nabla \alpha^{\prime}\left(\nabla \alpha^{\prime}\right)^{t}-\Phi^{\prime}\left(\alpha^{\prime}\right) \nabla^{2} \alpha^{\prime}\right\|_{\mathrm{L}^{2}(\Omega)} \\
& \leq\left\|\left(\Phi^{\prime \prime}(\alpha)-\Phi^{\prime \prime}\left(\alpha^{\prime}\right)\right) \nabla \alpha(\nabla \alpha)^{t}\right\|_{\mathrm{L}^{2}(\Omega)}+\left\|\Phi^{\prime \prime}\left(\alpha^{\prime}\right) \nabla\left(\alpha-\alpha^{\prime}\right)(\nabla \alpha)^{t}\right\|_{\mathrm{L}^{2}(\Omega)} \\
& +\left\|\Phi^{\prime \prime}\left(\alpha^{\prime}\right) \nabla \alpha^{\prime}\left(\nabla\left(\alpha-\alpha^{\prime}\right)\right)^{t}\right\|_{\mathrm{L}^{2}(\Omega)}+\left\|\Phi^{\prime}(\alpha) \nabla^{2}\left(\alpha-\alpha^{\prime}\right)\right\|_{\mathrm{L}^{2}(\Omega)} \\
& +\left\|\left(\Phi^{\prime}(\alpha)-\Phi^{\prime}\left(\alpha^{\prime}\right)\right) \nabla^{2} \alpha\right\|_{\mathrm{L}^{2}(\Omega)} \\
& \leq\left\|\Phi^{(3)}\right\|_{\mathrm{L}^{\infty}(\mathbb{R})}\left\|\alpha-\alpha^{\prime}\right\|_{\mathrm{L}^{\infty}}\|\nabla \alpha\|_{\mathrm{L}^{4}}\left\|\nabla \alpha^{\prime}\right\|_{\mathrm{L}^{4}}+\left\|\Phi^{\prime \prime}\right\|_{\mathrm{L}^{\infty}(\mathbb{R})}\left\|\nabla\left(\alpha-\alpha^{\prime}\right)\right\|_{\mathrm{L}^{4}}\|\nabla \alpha\|_{\mathrm{L}^{4}} \\
& +\left\|\Phi^{\prime \prime}\right\|_{\mathrm{L}^{\infty}(\mathbb{R})}\left\|\nabla\left(\alpha-\alpha^{\prime}\right)\right\|_{\mathrm{L}^{4}}\left\|\nabla \alpha^{\prime}\right\|_{\mathrm{L}^{4}}+\left\|\Phi^{\prime}\right\|_{\mathrm{L}^{\infty}(\mathbb{R})}\left\|\nabla^{2}\left(\alpha-\alpha^{\prime}\right)\right\|_{\mathrm{L}^{2}} \\
& +\left\|\Phi^{\prime \prime}\right\|_{\mathrm{L}^{\infty}(\mathbb{R})}\left\|\alpha-\alpha^{\prime}\right\|_{\mathrm{L}^{\infty}}\left\|\nabla^{2} \alpha^{\prime}\right\|_{\mathrm{L}^{2}} \\
& \leq C\|\Phi\|_{\mathrm{W}^{3, \infty}(\mathbb{R})}\left(\|\alpha\|_{\mathrm{H}^{2}(\Omega)}\left\|\alpha^{\prime}\right\|_{\mathrm{H}^{2}(\Omega)}+\|\alpha\|_{\mathrm{H}^{2}(\Omega)}+\left\|\alpha^{\prime}\right\|_{\mathrm{H}^{2}(\Omega)}+1\right)\left\|\alpha-\alpha^{\prime}\right\|_{\mathrm{H}^{2}(\Omega)} .
\end{aligned}
$$

Let $u$ and $u^{\prime}$ two solutions to (3.11) with $\mu$ and $\mu^{\prime}$ respectively.

Proposition 3.25. There exists a constant $C$ depending only on $\Omega$ such that for a.e. $t \in[0, T]$

$$
\left\|\left(u-u^{\prime}\right)(t, \cdot)\right\|_{H^{1}(\Omega)} \leq C\left\|u^{\prime}(t, \cdot)\right\|_{H^{2}(\Omega)}\left\|\left(\mu-\mu^{\prime}\right)(t, \cdot)\right\|_{H^{1}(\Omega)} \cdot
$$

Proof. The quantity $\left(u-u^{\prime}\right)$ fulfills

$$
\left\{\begin{array}{l}
-\operatorname{div}\left(2 \mu D\left(u-u^{\prime}\right)\right)-\operatorname{div}\left(2\left(\mu-\mu^{\prime}\right) D\left(u^{\prime}\right)\right)+\frac{\mathbf{1}_{B(t)}}{\varepsilon}\left(u-u^{\prime}\right)=-\nabla\left(p-p^{\prime}\right) \text { in }[0, T] \times \Omega, \\
\operatorname{div}\left(u-u^{\prime}\right)=0 \text { in }[0, T] \times \Omega \\
\left(u-u^{\prime}\right)=0 \text { on }[0, T] \times \Gamma_{0} \\
\left(u-u^{\prime}\right) \cdot \nu=\frac{\partial}{\partial \nu}\left(u-u^{\prime}\right)_{1}=\frac{\partial}{\partial \nu}\left(u-u^{\prime}\right)_{2}=0 \text { on }[0, T] \times \Gamma_{L} \\
\left(u-u^{\prime}\right) \text { has periodic conditions on }[0, T] \times \partial \Gamma \times[0, L] .
\end{array}\right.
$$

Taking the scalar product in $\mathrm{L}^{2}(\Omega)$ by $\left(u-u^{\prime}\right)$ for a.e. $t \in[0, T]$ we obtain thanks to Sobolev's injections

$$
\begin{aligned}
2 \int_{\Omega} \mu\left|D\left(u-u^{\prime}\right)\right|^{2} \mathrm{~d} x+\frac{1}{\varepsilon}\left\|u-u^{\prime}\right\|_{\mathrm{L}^{2}(B)}^{2} & =-\int_{\Omega} 2\left(\mu-\mu^{\prime}\right) D\left(u^{\prime}\right): D\left(u-u^{\prime}\right) \mathrm{d} x \\
& \leq 2\left\|\mu-\mu^{\prime}\right\|_{\mathrm{L}^{6}(\Omega)}\left\|D\left(u^{\prime}\right)\right\|_{\mathrm{L}^{3}(\Omega)}\left\|D\left(u-u^{\prime}\right)\right\|_{\mathrm{L}^{2}(\Omega)} \\
& \leq C\left\|\mu-\mu^{\prime}\right\|_{\mathrm{H}^{1}(\Omega)}\left\|u^{\prime}\right\|_{\mathrm{H}^{2}(\Omega)}\left\|D\left(u-u^{\prime}\right)\right\|_{\mathrm{L}^{2}(\Omega)} .
\end{aligned}
$$

This implies that there exists a constant $C$ depending only on $\Omega$ such that for a.e. $t \in[0, T]$,

$$
\left\|D\left(u-u^{\prime}\right)(t, \cdot)\right\|_{\mathrm{L}^{2}(\Omega)} \leq C\left\|u^{\prime}(t, \cdot)\right\|_{\mathrm{H}^{2}(\Omega)}\left\|\left(\mu-\mu^{\prime}\right)(t, \cdot)\right\|_{\mathrm{H}^{1}(\Omega)} .
$$


Let $\alpha$ and $\alpha^{\prime}$ two solutions to (3.5) respectively with $u$ and $u^{\prime}$.

Proposition 3.26. There exists a constant $C$ depending on $\Omega,\left\|\alpha_{0}\right\|_{H^{2}(\Omega)}, f, \bar{u},\|\mu\|_{L^{\infty}(\Omega)}$ such that for all $t \in[0, T]$

$$
\left\|\left(\alpha-\alpha^{\prime}\right)(t, \cdot)\right\|_{H^{1}(\Omega)}^{2} \leq C\left\|u-u^{\prime}\right\|_{L^{2}\left(0, t ; H^{1}(\Omega)\right)}^{2}
$$

and

$$
\left\|\alpha-\alpha^{\prime}\right\|_{L^{2}\left(0, t ; H^{2}(\Omega)\right)}^{2} \leq C\left\|u-u^{\prime}\right\|_{L^{2}\left(0, t ; H^{1}(\Omega)\right)}^{2} .
$$

Proof. The function $\beta=\alpha-\alpha^{\prime}$ fulfills:

$$
\left\{\begin{array}{l}
\partial_{t} \beta+u \cdot \nabla \beta-\eta \Delta \beta=-\left(u-u^{\prime}\right) \cdot \nabla \alpha^{\prime} \text { in } \Omega, \\
\frac{\partial \beta}{\partial \nu}=0 \text { on } \Gamma_{0} \cup \Gamma_{L}, \\
\beta \text { has periodic conditions on } \partial \Gamma \times[0, L], \\
\beta(0, \cdot)=0 \text { in } \Omega .
\end{array}\right.
$$

$\mathbf{L}^{2}$ estimate: we take the scalar product of (3.12) with $\beta$ in $\mathrm{L}^{2}(\Omega)$.

$$
\begin{aligned}
\frac{1}{2} \frac{\mathrm{d}}{\mathrm{d} t}\|\beta\|_{\mathrm{L}^{2}(\Omega)}^{2}+\eta\|\nabla \beta\|_{\mathrm{L}^{2}(\Omega)}^{2} & =-\int_{\Omega}\left(u-u^{\prime}\right) \cdot \nabla \alpha^{\prime} \beta \mathrm{d} x \\
& =\int_{\Omega^{\prime}}\left(u-u^{\prime}\right) \cdot \nabla \beta \alpha^{\prime} \mathrm{d} x \\
& \leq\left\|\alpha^{\prime}\right\|_{\mathrm{L}^{3}(\Omega)}\left\|u-u^{\prime}\right\|_{\mathrm{L}^{6}(\Omega)}\|\nabla \beta\|_{L^{2}(\Omega)} \\
& \leq C(\Omega)\left\|\alpha^{\prime}\right\|_{\mathrm{H}^{1}(\Omega)}\left\|u-u^{\prime}\right\|_{\mathrm{H}^{1}(\Omega)}\|\nabla \beta\|_{\mathrm{L}^{2}(\Omega)} \\
& \leq C(\Omega)\left\|\alpha^{\prime}\right\|_{\mathrm{H}^{1}(\Omega)}\left\|u-u^{\prime}\right\|_{\mathrm{H}^{1}(\Omega)}^{2}+\frac{\eta}{2}\|\nabla \beta\|_{\mathrm{L}^{2}(\Omega)}^{2} .
\end{aligned}
$$

$\boldsymbol{H}^{\mathbf{1}}$ estimate: we take the scalar product of (3.12) with $-\Delta \beta$ in $\mathrm{L}^{2}(\Omega)$.

$$
\begin{aligned}
\frac{1}{2} \frac{\mathrm{d}}{\mathrm{d} t}\|\nabla \beta\|_{\mathrm{L}^{2}(\Omega)}^{2}+\eta\|\Delta \beta\|_{\mathrm{L}^{2}(\Omega)}^{2}= & \int_{\Omega} u \cdot \nabla \beta \Delta \beta \mathrm{d} x+\int_{\Omega}\left(u-u^{\prime}\right) \cdot \nabla \alpha^{\prime} \Delta \beta \mathrm{d} x \\
\leq & \|u\|_{\mathrm{L}^{6}(\Omega)}\|\nabla \beta\|_{\mathrm{L}^{3}(\Omega)}\|\Delta \beta\|_{\mathrm{L}^{2}(\Omega)}+\left\|u-u^{\prime}\right\|_{\mathrm{L}^{6}(\Omega)}\left\|\nabla \alpha^{\prime}\right\|_{\mathrm{L}^{3}(\Omega)}\|\Delta \beta\|_{\mathrm{L}^{2}(\Omega)} \\
\leq & C(\Omega)\left(\|u\|_{\mathrm{H}^{1}(\Omega)}^{2}+\|u\|_{\mathrm{H}^{1}(\Omega)}^{4}\right)\|\beta\|_{\mathrm{H}^{1}(\Omega)}^{2}+C(\Omega)\left\|\alpha^{\prime}\right\|_{\mathrm{H}^{2}(\Omega)}^{2}\left\|u-u^{\prime}\right\|_{\mathrm{H}^{1}(\Omega)}^{2} \\
& +\frac{\eta}{2}\|\Delta \beta\|_{\mathrm{L}^{2}(\Omega)}^{2} .
\end{aligned}
$$

This implies that

$$
\frac{\mathrm{d}}{\mathrm{d} t}\|\beta\|_{\mathrm{H}^{1}(\Omega)}^{2}+\eta\left(\|\nabla \beta\|_{\mathrm{L}^{2}(\Omega)}^{2}+\|\Delta \beta\|_{\mathrm{L}^{2}(\Omega)}^{2}\right) \leq C(\Omega)\left(\|u\|_{\mathrm{H}^{1}(\Omega)}^{2}+\|u\|_{\mathrm{H}^{1}(\Omega)}^{4}\right)\|\beta\|_{\mathrm{H}^{1}(\Omega)}^{2}+C(\Omega)\left\|\alpha^{\prime}\right\|_{\mathrm{H}^{2}(\Omega)}\left\|u-u^{\prime}\right\|_{\mathrm{H}^{1}(\Omega)}^{2}
$$

We let $y=\|\beta\|_{\mathrm{H}^{1}(\Omega)}^{2}$ and $y_{1}=\|\nabla \beta\|_{\mathrm{L}^{2}(\Omega)}^{2}+\|\Delta \beta\|_{\mathrm{L}^{2}(\Omega)}^{2}$. Thanks to the regularity results on $u$, there exists $k \in \mathrm{L}^{1}(0, T)$ and a constant $C$ depending only on $\Omega,\left\|\alpha_{0}\right\|_{\mathrm{H}^{2}(\Omega)}, f, \bar{u},\|\Phi\|_{\mathrm{L}^{\infty}(\mathbb{R})}$ such that for all $0 \leq t \leq T$,

$$
y^{\prime}+\eta y_{1} \leq k(t) y+C\left\|u-u^{\prime}\right\|_{\mathrm{H}^{1}(\Omega)}^{2} .
$$

Since $y(0)=0$, we obtain thanks to Gronwall's lemma that for all $t \in[0, T]$,

$$
\begin{aligned}
y(t)+\eta \int_{0}^{t} y_{1}(s) \mathrm{d} s & \leq \int_{0}^{t} C\left\|\left(u-u^{\prime}\right)(s, \cdot)\right\|_{\mathrm{H}^{1}(\Omega)}^{2} \exp \left(\int_{s}^{t} k\left(s^{\prime}\right) \mathrm{d} s^{\prime}\right) \mathrm{d} s \\
& \leq C \exp \left(\|k\|_{\mathrm{L}^{1}(0, T)}\right)\left\|u-u^{\prime}\right\|_{\mathrm{L}^{2}\left(0, t ; H^{1}(\Omega)\right)}^{2} \\
& \leq C^{\prime}\left\|u-u^{\prime}\right\|_{\mathrm{L}^{2}\left(0, t ; H^{1}(\Omega)\right)}^{2} .
\end{aligned}
$$


Proposition 3.27. There exists a time $T^{*}$ depending only on $T, \Omega, O_{p},\left\|\alpha_{0}\right\|_{H^{2}(\Omega)}, f, \bar{u}$ and $\Phi$ such that

$$
\left\|G(\alpha)-G\left(\alpha^{\prime}\right)\right\|_{L^{\infty}\left(0, T^{*} ; H^{1}(\Omega)\right)} \leq \frac{1}{2}\left\|\alpha-\alpha^{\prime}\right\|_{L^{\infty}\left(0, T^{*} ; H^{1}(\Omega)\right)} .
$$

Proof. This result is the consequence of Propositions 3.24, 3.25 and 3.26: for all $t \in[0, T]$ we have

$$
\begin{aligned}
\left\|\left(G(\alpha)-G\left(\alpha^{\prime}\right)\right)(t, \cdot)\right\|_{\mathrm{H}^{1}(\Omega)} & \leq C\left\|u-u^{\prime}\right\|_{\mathrm{L}^{2}\left(0, t ; H^{1}(\Omega)\right)} \\
& \leq C\left(\int_{0}^{t}\left\|u^{\prime}(t, \cdot)\right\|_{\mathrm{H}^{2}(\Omega)}^{2}\left\|\left(\mu-\mu^{\prime}\right)(s, \cdot)\right\|_{\mathrm{H}^{1}(\Omega)}^{2} \mathrm{~d} s\right)^{1 / 2} \\
& \leq C\left\|u^{\prime}\right\|_{\mathrm{L}^{2}\left(0, t ; \mathrm{H}^{2}(\Omega)\right)}\left\|\mu-\mu^{\prime}\right\|_{\mathrm{L}^{\infty}\left(0, t ; \mathrm{H}^{1}(\Omega)\right)}\left\|C\left(1+\left\|\alpha^{\prime}\right\|_{\mathrm{L}^{\infty}\left(0, T ; \mathrm{H}^{2}(\Omega)\right)}\right)\right\| u^{\prime}\left\|_{\mathrm{L}^{2}\left(0, t ; \mathrm{H}^{2}(\Omega)\right)}\right\| \alpha-\alpha^{\prime} \|_{\mathrm{L}^{\infty}\left(0, t ; \mathrm{H}^{1}(\Omega)\right)} .
\end{aligned}
$$

Thanks to Theorems 3.22 and $3.21, \alpha^{\prime}$ is bounded in $\mathrm{L}^{\infty}\left(0, T ; \mathrm{H}^{2}(\Omega)\right)$ and in $\mathrm{L}^{2}\left(0, t ; \mathrm{H}^{3}(\Omega)\right)$ for all $0 \leq t \leq T$. Then $\mu^{\prime} \in \mathrm{L}^{\infty}([0, T] \times \Omega)$ also fulfills

$$
\begin{aligned}
\left\|\nabla \mu^{\prime}(t, \cdot)\right\|_{\mathrm{L}^{\infty}(\Omega)} & \leq\left\|\Phi^{\prime}\right\|_{\mathrm{L}^{\infty}(\mathbb{R})}\|\nabla \alpha(t, \cdot)\|_{\mathrm{L}^{\infty}(\Omega)} \\
& \leq C\left\|\Phi^{\prime}\right\|_{\mathrm{L}^{\infty}(\mathbb{R})}\|\nabla \alpha(t, \cdot)\|_{\mathrm{H}^{1}(\Omega)}^{114}\|\nabla \alpha(t, \cdot)\|_{\mathrm{H}^{2}(\Omega)}^{3 / 4} \\
& \leq C\left\|\Phi^{\prime}\right\|_{\mathrm{L}^{\infty}(\mathbb{R})}\|\alpha(t, \cdot)\|_{\mathrm{H}^{2}(\Omega)}^{1 / 4}\|\alpha(t, \cdot)\|_{\mathrm{H}^{3}(\Omega)}^{3 / 4} .
\end{aligned}
$$

This implies that $\mu^{\prime} \in \mathrm{L}^{8 / 3}\left(0, T ; \mathrm{W}^{1, \infty}(\Omega)\right)$ and, thanks to Theorem $3.21, u^{\prime}$ is bounded in $\mathrm{L}^{8 / 3}\left(0, T ; \mathrm{H}^{2}(\Omega)\right)$ and

$$
\left\|u^{\prime}\right\|_{\mathrm{L}^{2}\left(0, t ; \mathrm{H}^{2}(\Omega)\right)} \leq t^{1 / 8}\left\|u^{\prime}\right\|_{\mathrm{L}^{8 / 3}\left(0, T ; \mathrm{H}^{2}(\Omega)\right)} .
$$

Then there exists a time $0<T^{*}<T$ such that for all $0 \leq t \leq T^{*}$,

$$
\left\|\left(G(\alpha)-G\left(\alpha^{\prime}\right)\right)(t, \cdot)\right\|_{\mathrm{H}^{1}(\Omega)} \leq \frac{1}{2}\left\|\alpha-\alpha^{\prime}\right\|_{\mathrm{L}^{\infty}\left(0, T^{*} ; \mathrm{H}^{1}(\Omega)\right)} .
$$

Moreover $T^{*}$ depends on $T, \Omega, O_{p}, f, \bar{u}, \alpha_{0},\|\Phi\|_{\mathrm{L}^{\infty}(\mathbb{R})}$ and $\varepsilon$.

\subsubsection{Proof of Theorem 3.5}

The sequence $\left(\alpha^{n}\right)_{n \in \mathbb{N}}$ defined in Section 3.6 is bounded in $\mathrm{L}^{\infty}\left(0, T ; \mathrm{H}^{2}(\Omega)\right) \cap \mathrm{L}^{2}\left(0, T ; \mathrm{H}^{3}(\Omega)\right) \cap$ $\mathrm{H}^{1}\left(0, T ; \mathrm{H}^{1}(\Omega)\right)$, as well as $\left(u^{n}\right)_{n \in \mathbb{N}}$ in $\mathrm{L}^{\infty}(0, T ; V(\Omega)), p^{n} \in \mathrm{L}^{2}\left(0, T ; \mathrm{L}_{0}^{2}(\Omega)\right)$ and $\left(\mu^{n}\right)_{n \in \mathbb{N}}$ in $\mathrm{L}^{\infty}\left(\left(0, T ; \mathrm{H}^{1}(\Omega)\right)\right.$.

The application $G$ is a contraction on $\mathrm{L}^{\infty}\left(0, T^{*} ; \mathrm{H}^{1}(\Omega)\right)$. This implies that the recurring sequence $\left(\alpha^{n}\right)_{n \in \mathbb{N}}$ defined in Section 3.6 converges strongly in $\mathrm{L}^{\infty}\left(0, T^{*} ; \mathrm{H}^{1}(\Omega)\right)$, and thanks to Propositions 3.24 and 3.25, the sequences $\left(\mu^{n}\right)_{n \in \mathbb{N}}$ and $\left(u^{n}\right)_{n \in \mathbb{N}}$ are Cauchy sequences and converge strongly towards $\mu \in \mathrm{L}^{\infty}\left(0, T^{*} ; \mathrm{H}^{1}(\Omega)\right)$ and $u \in \mathrm{L}^{\infty}\left(0, T^{*} ; V(\Omega)\right)$ respectively.

Thanks to the boundedness of the sequences we moreover have that there exists a subsequence of $\left(\alpha^{n}\right)_{n \in \mathbb{N}}$ and $\left(p^{n}\right)_{n \in \mathbb{N}}$ still denoted $\left(\alpha^{n}\right)_{n \in \mathbb{N}}$ and $\left(p^{n}\right)_{n \in \mathbb{N}}$ that fulfill:

$$
\begin{aligned}
& \alpha^{n} \rightarrow \alpha \text { weak }-\star \text { in } \mathrm{L}^{\infty}\left(0, T^{*} ; \mathrm{H}^{2}(\Omega)\right), \\
& \alpha^{n} \rightarrow \alpha \text { weak in } \mathrm{L}^{2}\left(0, T^{*} ; \mathrm{H}^{3}(\Omega)\right), \\
& \alpha^{n} \rightarrow \alpha \text { weak in } \mathrm{H}^{1}\left(0, T^{*} ; \mathrm{H}^{1}(\Omega)\right), \\
& p^{n} \rightarrow p \quad \text { weak in } \mathrm{L}^{2}\left(0, T^{*} ; \mathrm{L}_{0}^{2}(\Omega)\right) .
\end{aligned}
$$

We now take the limit as $n$ goes to $+\infty$ in the sequence defined in 3.6 and obtain that $(u, p, \alpha)$ is a weak solution to $(3.3)$ on $\left(0, T^{*}\right) \times \Omega$.

Moreover the time $T^{*}$ depends only on the data of the problem, particularly through the estimate on $\alpha \in$ $\mathrm{L}^{\infty}\left(0, T ; \mathrm{H}^{2}(\Omega)\right)$ obtained in Theorem 3.22

$$
\|\alpha\|_{\mathrm{L}^{\infty}\left(0, T ; \mathrm{H}^{2}(\Omega)\right)} \leq C\left\|\alpha_{0}\right\|_{\mathrm{H}^{2}(\Omega)} \exp \left(C^{\prime} \int_{0}^{T}\left(1+\|u(s, \cdot)\|_{\mathrm{H}^{1}(\Omega)}^{2}+\|u(s, \cdot)\|_{\mathrm{H}^{1}(\Omega)}^{4}+\|u(s, \cdot)\|_{\mathrm{H}^{1}(\Omega)}^{8}\right) \mathrm{d} s\right),
$$


with estimates on $u$ only depending on the data of the problem (Thm. 3.21). We now bound $\left\|\alpha_{0}\right\|_{H^{2}(\Omega)}$ with this estimate and take a smaller $T^{*}$ to fulfill our previous constraints. Since $\alpha \in \mathrm{L}^{2}\left(0, T ; H^{3}(\Omega)\right)$ and $\frac{\partial \alpha}{\partial t} \in$ $\mathrm{L}^{2}\left(0, T ; \mathrm{H}^{1}(\Omega)\right)$ then $\alpha \in \mathcal{C}\left(\left[0, T^{*}\right] ; \mathrm{H}^{2}(\Omega)\right)\left(c f\right.$. Thm. 3.11). This implies that $\alpha(0, \cdot)=\alpha_{0} \in \mathrm{H}^{2}(\Omega)$ and that $\alpha\left(T^{*}, \cdot\right) \in \mathrm{H}^{2}(\Omega)$. $\left\|\alpha\left(T^{*}, \cdot\right)\right\|_{\mathrm{H}^{2}(\Omega)}$ also fulfills the same bound as $\left\|\alpha_{0}\right\|_{\mathrm{H}^{2}(\Omega)}$. We now apply the same estimates starting from $T^{*}$ and obtain the existence and the uniqueness of the solution on $\left(T^{*}, 2 T^{*}\right)$ and finally on $(0, T)$.

\subsection{Proof of Theorem 3.7}

According to Theorem 3.5 we have that

- $\alpha^{\varepsilon}$ is bounded independently from $\varepsilon$ in the spaces $\mathrm{L}^{\infty}\left(0, T ; \mathrm{H}^{2}(\Omega)\right), \mathrm{L}^{2}\left(0, T ; \mathrm{H}^{3}(\Omega)\right)$ and $\mathrm{H}^{1}\left(0, T ; \mathrm{H}^{1}(\Omega)\right)$, which also implies that $\alpha^{\varepsilon}$ and $\nabla \alpha^{\varepsilon}$ are bounded in $\mathrm{H}^{1}((0, T) \times \Omega)$,

- $u^{\varepsilon}$ is bounded in $\mathrm{L}^{q}(0, T ; V(\Omega))$ for all $2 \leq q \leq+\infty$,

- $\varepsilon^{-1}\left\|u^{\varepsilon}-\bar{u}\right\|_{\mathrm{L}^{q}(0, T ; B(\cdot))}^{2}$ is bounded for all $2 \leq q \leq+\infty$.

There exists then a subsequence still denoted $\left(u^{\varepsilon}, p^{\varepsilon}, \alpha^{\varepsilon}\right)$ that fulfills

$$
\begin{array}{cl}
\alpha^{\varepsilon} \rightarrow \alpha & \text { weak }-\star \text { in } \mathrm{L}^{\infty}\left(0, T ; \mathrm{H}^{2}(\Omega)\right), \\
\alpha^{\varepsilon} \rightarrow \alpha & \text { weak in } \mathrm{L}^{2}\left(0, T ; \mathrm{H}^{3}(\Omega)\right), \\
\alpha^{\varepsilon} \rightarrow \alpha & \text { weak in } \mathrm{H}^{1}\left(0, T ; \mathrm{H}^{1}(\Omega)\right), \\
\alpha^{\varepsilon} \rightarrow \alpha & \text { strong in } \mathrm{L}^{q}((0, T) \times \Omega) \text { for all } 2 \leq q \leq 4, \\
\nabla \alpha^{\varepsilon} \rightarrow \nabla \alpha & \text { strong in } \mathrm{L}^{q}((0, T) \times \Omega) \text { for all } 2 \leq q \leq 4, \\
u^{\varepsilon} \rightarrow u & \text { weak in } \mathrm{L}^{q}(0, T ; V(\Omega)) \text { for all } 2 \leq q<+\infty, \\
u^{\varepsilon} \rightarrow u & \text { weak }-\star \text { in } \mathrm{L}^{\infty}(0, T ; V(\Omega)), \\
u^{\varepsilon} \rightarrow \bar{u} & \text { strong in } \mathrm{L}^{q}\left(0, T ; \mathrm{L}^{2}(B(\cdot))\right) \text { for all } 2 \leq q \leq+\infty,
\end{array}
$$

Study of $\alpha$ : Thanks to the strong convergence of $\nabla \alpha^{\varepsilon}$ in $\mathrm{L}^{4}((0, T) \times \Omega)$ and the weak convergence of $u^{\varepsilon}$ in $\mathrm{L}^{4}((0, T) \times \Omega) u^{\varepsilon} \cdot \nabla \alpha^{\varepsilon}$ converges weakly toward $u \cdot \nabla \alpha$ in $\mathrm{L}^{2}((0, T) \times \Omega)$. This implies that we can take the limit in the weak formulation of the convection-diffusion equation.

Since $\alpha^{\varepsilon}$ converges weakly towards $\alpha$ in $\mathrm{H}^{1}\left(0, T ; \mathrm{H}^{1}(\Omega)\right), \alpha^{\varepsilon}(0, \cdot)$ converges weakly towards $\alpha(0, \cdot)$ in $\mathrm{H}^{1}(\Omega)$ which implies that $\alpha(0, \cdot)=\alpha_{0} \in \mathrm{H}^{2}(\Omega)$ in the trace sense.

Study of $\mu$ : We are now interested in the convergence of $\mu^{\varepsilon}=\Phi\left(\alpha^{\varepsilon}\right)$. Thanks to the strong convergence of $\alpha^{\varepsilon}$ towards $\alpha$ in $\mathrm{L}^{4}((0, T) \times \Omega), \mu^{\varepsilon}$ converges strongly towards $\mu=\Phi(\alpha)$ in $\mathrm{L}^{4}((0, T) \times \Omega)$. Moreover using the boundedness of $\alpha^{\varepsilon}$ we obtain that $\mu^{\varepsilon}$ and $\nabla \mu^{\varepsilon}$ are bounded in $\mathrm{H}^{1}((0, T) \times \Omega)$. This implies that $\mu^{\varepsilon}$ converges strongly towards $\mu$ in $\mathrm{L}^{4}\left(0, T ; \mathrm{W}^{1,4}(\Omega)\right)$ and then in $\mathrm{L}^{4}\left(0, T ; \mathrm{L}^{\infty}(\Omega)\right)$.

Study of $(\boldsymbol{u}$ et $\boldsymbol{p})$ : Thanks to the weak convergence of $u^{\varepsilon}$ towards $u$ in $L^{2}(0, T ; V(\Omega))$ and to the strong convergence of $u^{\varepsilon}$ towards $\bar{u}$ in $\mathrm{L}^{2}\left(O_{p}\right)$, we have $u=\bar{u}$ in $O_{p}$.

In $O_{p}^{c},\left(u^{\varepsilon}, p^{\varepsilon}\right)$ is a weak solution to

$$
\left\{\begin{array}{l}
-\operatorname{div}\left(2 \mu^{\varepsilon} D\left(u^{\varepsilon}\right)\right)=f-\nabla p^{\varepsilon} \quad \text { in } O_{p}^{c}, \\
\operatorname{div} u^{\varepsilon}=0 \text { in } O_{p}^{c}, \\
u^{\varepsilon} \cdot \nu=\frac{\partial u_{1}^{\varepsilon}}{\partial \nu}=\frac{\partial u_{2}^{\varepsilon}}{\partial \nu}=0 \quad \text { on }[0, T] \times \Gamma_{L}, \\
u^{\varepsilon} \text { has periodic conditions on }[0, T] \times \partial \Gamma \times[l, L] .
\end{array}\right.
$$

Since $\mu^{\varepsilon}=\Phi\left(\alpha^{\varepsilon}\right)$ converges strongly towards $\mu=\Phi(\alpha)$ in $\mathrm{L}^{4}\left(0, T ; \mathrm{L}^{\infty}(\Omega)\right)$ and thanks to the weak convergence of $u^{\varepsilon}$ towards $u$ in $\mathrm{L}^{4}\left(0, T ; H^{1}(\Omega \backslash \bar{B}(\cdot))\right), 2 \mu^{\varepsilon} D\left(u^{\varepsilon}\right)$ converges weakly towards $2 \mu D(u)$ in $\mathrm{L}^{2}\left(O_{p}^{c}\right)$. This also implies that $\nabla p^{\varepsilon}$ converges weakly towards $\nabla p$ in $\mathrm{L}^{2}\left(0, T ; \mathrm{H}^{-1}(\Omega \backslash \bar{B}(\cdot))\right)$ and then the weak convergence of $p^{\varepsilon}$ towards $p$ in $\mathrm{L}^{2}\left(0, T ; \mathrm{L}_{0}^{2}(\Omega \backslash \bar{B}(\cdot))\right)$. 
Thanks to the weak convergence of $u^{\varepsilon}$ towards $u$ in $\mathrm{L}^{q}(0, T ; V(\Omega))$ for all $2 \leq q \leq+\infty$, we deduce that $(u, p)$ fulfills

$$
\left\{\begin{array}{l}
-\operatorname{div}(2 \mu D(u))=f-\nabla p \text { in } O_{p}^{c}, \\
\operatorname{div} u=0 \text { in } O_{p}^{c} \\
u \cdot \nu=\frac{\partial u_{1}}{\partial \nu}=\frac{\partial u_{2}}{\partial \nu}=0 \quad \text { on }[0, T] \times \Gamma_{L}, \\
u \text { has periodic conditions on }[0, T] \times \partial \Gamma \times[0, L] .
\end{array}\right.
$$

Since $u \in L^{2}(0, T ; V(\Omega)) \subset \mathrm{L}^{2}\left(0, T ; \mathrm{H}^{1}(\Omega)\right)$ then $u_{\mid \partial B(\cdot) \cap \Omega} \in \mathrm{L}^{2}\left(0, T ; \mathrm{H}^{1 / 2}(\partial B(\cdot) \cap \Omega)\right)$. Moreover $u=\bar{u}$ in $O_{p}$ with $\bar{u} \in \mathrm{H}^{3}\left(O_{p}\right)$. Then $u_{\mid \partial B(\cdot) \cap \Omega}=\bar{u}_{\mid \partial B(\cdot) \cap \Omega}$ in $\mathrm{L}^{2}\left(0, T ; \mathrm{H}^{1 / 2}(\partial B(\cdot) \cap \Omega)\right.$.

Uniqueness: Let $(u, p, \alpha)$ and $\left(u^{\prime}, p^{\prime}, \alpha^{\prime}\right)$ two weak solutions to (3.1). The inequalities we obtained on $\alpha-\alpha^{\prime}$ in Proposition 3.26 remain valid: for all $t \in[0, T]$,

$$
\begin{aligned}
& \left\|\left(\alpha-\alpha^{\prime}\right)(t, \cdot)\right\|_{\mathrm{H}^{1}(\Omega)} \leq C\left\|u-u^{\prime}\right\|_{\mathrm{L}^{2}\left(0, t ; \mathrm{H}^{1}(\Omega)\right)} \\
& \left\|\alpha-\alpha^{\prime}\right\|_{\mathrm{L}^{2}\left(0, t ; \mathrm{H}^{2}(\Omega)\right)} \leq C\left\|u-u^{\prime}\right\|_{\mathrm{L}^{2}\left(0, t ; \mathrm{H}^{1}(\Omega)\right)}
\end{aligned}
$$

Following the estimates in Proposition 3.25 we write for a.e. $t \in[0, T]$,

$$
\int_{\Omega \backslash \bar{B}(t)} 2 \mu\left|D\left(u-u^{\prime}\right)\right|^{2}(t, x) \mathrm{d} x \leq C\left\|\mu-\mu^{\prime}\right\|_{\mathrm{L}^{\infty}(\Omega \backslash \bar{B}(t))}\left\|u^{\prime}\right\|_{\mathrm{H}^{1}(\Omega \backslash \bar{B}(t))}\left\|D\left(u-u^{\prime}\right)\right\|_{\mathrm{H}^{1}(\Omega \backslash \bar{B}(t))} .
$$

Since $u-u^{\prime}=0$ on $B(t)$ for a.e. $t \in[0, T]$ we obtain thanks to Lemma 3.9

$$
\begin{aligned}
\left\|D\left(u-u^{\prime}\right)(t, \cdot)\right\|_{\mathrm{L}^{2}(\Omega)} & \leq C\left\|u^{\prime}\right\|_{\mathrm{H}^{1}(\Omega)}\left\|\mu-\mu^{\prime}\right\|_{\mathrm{L}^{\infty}(\Omega)} \\
& \leq C\left\|u^{\prime}\right\|_{\mathrm{H}^{1}(\Omega)}\left\|\mu-\mu^{\prime}\right\|_{\mathrm{H}^{1}(\Omega)}^{1 / 4}\left\|\mu-\mu^{\prime}\right\|_{\mathrm{H}^{2}(\Omega)}^{3 / 4} .
\end{aligned}
$$

Thanks to the bounds on $u, u^{\prime}, \alpha$ and $\alpha^{\prime}$ and to Proposition 3.24 we obtain for a.e. $t \in[0, T]$,

$$
\left\|D\left(u-u^{\prime}\right)(t, \cdot)\right\|_{\mathrm{L}^{2}(\Omega)} \leq C\left\|\left(\alpha-\alpha^{\prime}\right)(t, \cdot)\right\|_{\mathrm{H}^{1}(\Omega)}^{1 / 4}\left\|\left(\alpha-\alpha^{\prime}\right)(t, \cdot)\right\|_{\mathrm{H}^{2}(\Omega)}^{3 / 4} .
$$

Combining all these inequalities we obtain that

$$
\begin{aligned}
\left\|u-u^{\prime}\right\|_{\mathrm{L}^{2}\left(0, t ; \mathrm{H}^{1}(\Omega)\right)}^{2} & \leq C \int_{0}^{t}\left\|\left(\alpha-\alpha^{\prime}\right)(s, \cdot)\right\|_{\mathrm{H}^{1}(\Omega)}^{1 / 2}\left\|\left(\alpha-\alpha^{\prime}\right)(s, \cdot)\right\|_{\mathrm{H}^{2}(\Omega)}^{3 / 2} \mathrm{~d} s \\
& \leq C\left(\int_{0}^{t}\left\|\left(\alpha-\alpha^{\prime}\right)(s, \cdot)\right\|_{\mathrm{H}^{1}(\Omega)}^{2} \mathrm{~d} s\right)^{1 / 4}\left\|\alpha-\alpha^{\prime}\right\|_{\mathrm{L}^{2}\left(0, t ; \mathrm{H}^{2}(\Omega)\right)}^{3 / 2} \\
& \leq C\left(\int_{0}^{t}\left\|\left(\alpha-\alpha^{\prime}\right)(s, \cdot)\right\|_{\mathrm{H}^{1}(\Omega)}^{2} \mathrm{~d} s\right)^{1 / 4}\left\|u-u^{\prime}\right\|_{\mathrm{L}^{2}\left(0, t ; \mathrm{H}^{1}(\Omega)\right)}^{3 / 2}
\end{aligned}
$$

Hence

$$
\left\|u-u^{\prime}\right\|_{\mathrm{L}^{2}\left(0, t ; \mathrm{H}^{1}(\Omega)\right)}^{2} \leq C \int_{0}^{t}\left\|\left(\alpha-\alpha^{\prime}\right)(s, \cdot)\right\|_{\mathrm{H}^{1}(\Omega)}^{2} \mathrm{~d} s
$$

and

$$
\left\|\left(\alpha-\alpha^{\prime}\right)(t, \cdot)\right\|_{\mathrm{H}^{1}(\Omega)}^{2} \leq C\left\|u-u^{\prime}\right\|_{L^{2}\left(0, t ; \mathrm{H}^{1}(\Omega)\right)}^{2} \leq C \int_{0}^{t}\left\|\left(\alpha-\alpha^{\prime}\right)(s, \cdot)\right\|_{\mathrm{H}^{1}(\Omega)}^{2} \mathrm{~d} s .
$$

Thanks to Gronwall lemma we have for all $t \in[0, T]$

$$
\left\|\left(\alpha-\alpha^{\prime}\right)(t, \cdot)\right\|_{\mathrm{H}^{1}(\Omega)}=0,
$$

and then $u=u^{\prime}$ and $p=p^{\prime}$. 


\section{Numerical Applications}

An original algorithm has been developed to compute numerically the solution to the penalized Stokes problem coupled to the convection-diffusion of the mass fraction. After a brief presentation of discretization techniques and computational principles, two highly viscous flows are presented.

The first one is a porous media flow at the scale of the pores. This simulation was performed under the assumption of a weak coupling between mass fraction $\alpha$ and velocity $u$ (with $\Phi=\mu_{\text {water }}$, the water's viscosity). It shows that the penalty method is a meaningful formulation to use with such complex geometries.

The second simulation concerns biomedical applications: the mucus flow in the lung at the scale of ciliated epithelium cells. In this second application the full coupling is assumed, as described in the previous sections, with moving and deformable obstacles (the cilia) and a variable viscosity fluid.

Although in this section the velocity is computed using the penalty method, the velocity is still denoted $u$, omitting the $\varepsilon$ subscripts.

\subsection{Discretization}

As the penalty method performs a coupling between solid bodies and the surrounded fluid through the characteristic function $\mathbf{1}_{B(t)}$, a precise mesh of the solid domain is not needed. Cartesian grids are a good framework for computations since this grid regularity is a great benefit for large 3D problems. Numerical applications are computed with a fast algorithm developed with a hybrid grid/particles approach.

After using Lagrangian and splitting techniques (see $[11,13,15,20]$ for details), the convection of $\alpha$ is solved using a particle discretization. It transforms the convection partial differential equation into a set of ordinary differential equations posed on particles. Particles are pushed along characteristic curves defined by the velocity field, which is the solution to the Stokes problem. Thus the related stability condition vanishes and large time steps can be performed without loosing significant accuracy, since constants in error estimates are small.

The accuracy of the method is guaranteed by high order non diffusive interpolation kernels between the grid and particles. The diffusion of $\alpha$ is explicitly computed on the grid with standard finite difference stencils for the heat equation. It does not limit the method since $\eta$ is small enough to be under the stability condition threshold. With these Lagrangian techniques the global cost of the computations is linear and the key point is to compute the velocity field, solution to the Stokes problem, without ruining this accuracy.

The Stokes problem is computed in an Eulerian way on a Cartesian grid. A projection method is used to split the velocity and the pressure computations (linked by the incompressibility condition). The projection of the $3 \mathrm{D}$ field $u^{*}$ is performed with the following correction:

$$
u=\Pi\left(u^{*}\right)=u^{*}-\nabla \zeta
$$

such that the projected solution $u$ is divergence free. The projector $\zeta$ is the solution to the following Poisson problem:

$$
\left\{\begin{array}{l}
-\Delta \zeta=-\operatorname{div} u^{*} \\
\frac{\partial \zeta}{\partial n}=u^{*} \cdot n \quad \text { on boundary. }
\end{array}\right.
$$

A variant of Chorin's original projection method is adapted for this quasi-static problem using a fixed point method. It consists in using the projector gradient computed at the previous iteration to guarantee both the incompressibility and an accurate tangential boundary condition. The method is decomposed in two steps (in the following, the index $k$ denotes the iteration number): in the first step, an intermediate velocity field $u_{k+1}^{*}$ is computed as the solution to the following modified stokes problem:

$$
\left\{\begin{array}{l}
-\mu \Delta u_{k+1}^{*}+\frac{\mathbf{1}_{B(t)}}{\varepsilon}\left(u_{k+1}^{*}-\nabla \zeta_{k}-\bar{u}\right)=f+2 D\left(u_{k}\right) \nabla \mu+\operatorname{div} u_{k}^{*} \nabla \mu, \\
u_{k+1}^{*}=g+\nabla \zeta_{k} \quad \text { on boundary. }
\end{array}\right.
$$


with the extra term $\operatorname{div} u_{k}^{*} \nabla \mu$ coming from the substitution of $u_{k}=u_{k}^{*}-\nabla \zeta_{k}$ in the original Stokes problem. This intermediate velocity field in then projected using the operator $\Pi$ introduced above.

This iterative process is also embedding extra terms resulting from the non homogeneity of the viscosity. They are treated explicitly, so the computation of the three velocity field components remains decoupled. Thus the computational cost for a variable or constant viscosity fluid is similar.

The limit of this sequence is then solution of the penalized variable viscosity 3D Stokes equation [11]:

$$
\left\{\begin{array}{l}
-\operatorname{div}\left(\mu\left(\nabla u+\nabla u^{T}\right)\right)+\frac{\mathbf{1}_{B(t)}}{\varepsilon}(u-\bar{u})+\nabla p=f, \\
u=g \text { on boundary. }
\end{array}\right.
$$

This fractional step algorithm transforms the full Stokes problem into a sequence of Poisson and Helmholtz problems with space varying coefficients. For these well-known elliptic problems, fast solvers can be used. The stiffness of coefficients in the Helmholtz equation can be difficult to handle straightly and an alternative is to use an iterative GMRES method coupled with a Sherman-Morrison-Woodbury approach [12]. It is particularly reliable, even if the penalized domain is composed of several different objects. In this work MUDPACK and FISHPACK elliptic solvers are used $[1,41,42]$. The first one uses a multi-grid approach and the second one is based on Fast Fourier Transforms and Tchebychev polynomial decompositions.

With this algorithm, standard convergence rates were numerically validated: it has a first order accuracy with respect to the spatial discretization [33] and a $\frac{1}{2}$ convergence rate with respect to the penalty parameter $\varepsilon$ [9]. These numerical validations can be found in $[10,11]$ as well as quasi-linear global computational cost (which is a consequence of the use of fast FFT and multi-grid solvers).

\subsection{Application to porous media}

The first illustration concerns porous media flows. In this computation the fluid flows in a network of silica micro-spheres. It is driven by a vertical constant external force at RHS of the momentum conservation equation. For this application the viscosity is assumed to be constant (equal to the viscosity of water) and $\alpha$ is a passive tracer in the flow. It means that the velocity field $u$ is computed once and $\alpha$ is then convected in the pores network. It is initialized constant (equal to one) at four locations on the top of the computational box, over 4 space discretization intervals; its value is zero in the rest of the computational domain. This kind of flow arises in many applications: medical modeling (drug delivering through skin pores, protein secretions, ...), ecology phenomena (water/pollution infiltration, tree roots ...), process or mechanical engineering (polymer, concretes ...).

This flow is very interesting to test the robustness of our algorithm since the solid characteristic function $\mathbf{1}_{B}$ is very complex - see Figure 2. Computations are performed on a $1024^{3}$ grid over 5000 time steps. The temporal integration of the Lagrangian ODE is performed with an Adams Bashforth 3 scheme.

Different snapshots of an isosurface of $\alpha$ are presented on Figure 2. The passive tracer is convected inside the pore network, showing the connectedness of the network. The high resolution of the refinement is justified by the characteristic size of emerging structures. It is then possible to quantify how long it takes for the passive tracer to reach the bottom of the computational box. This time has to be shorten as much as possible for drug delivering applications while it must be long if quantifying pollution infiltrations through drinkable water reserves for example.

Finally this simulation enables the computation of the porosity tensor $\boldsymbol{K}$ associated to the geometry. This tensor is used to compute the velocity field resulting to a driving pressure $\nabla \pi=f$ or $\nabla p$ with the Darcy equation. It is an intrinsic geological parameter of rock formation, obtained by averaging velocity and driving pressure on the domain or on a part of it:

$$
\langle u\rangle=\mu^{-1} \boldsymbol{K}\langle\nabla \pi\rangle .
$$

This holds for constant viscosity $\mu$ and it is a consequence of Stokes problem linearity. In this equation $\boldsymbol{K}$ is a $3 \times 3$ tensor. The first column of each tensor is computed using our Stokes solver with a force $\nabla \pi$ equals to the 

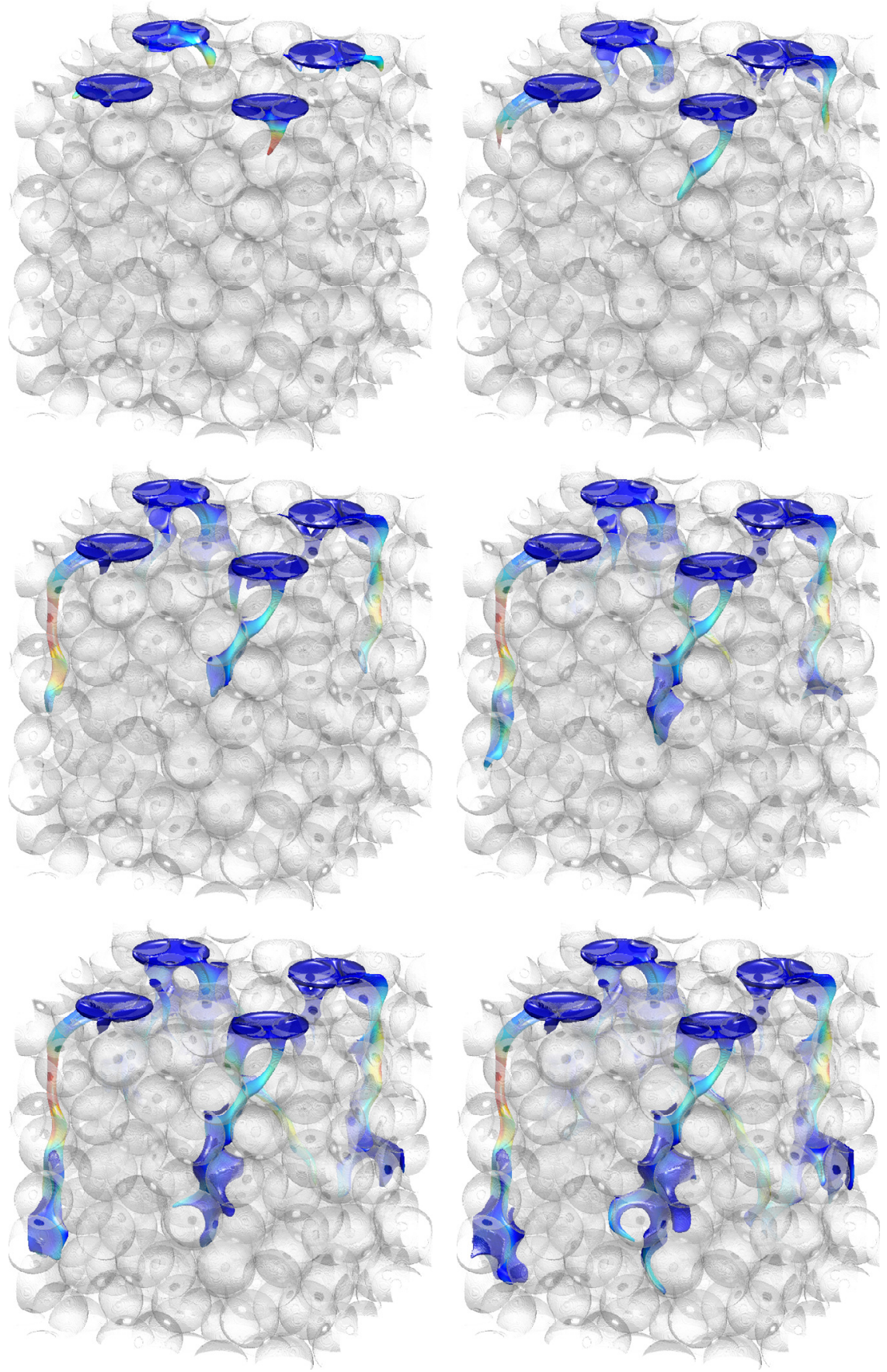

Figure 2. Different snapshots of an isosurface of the passive tracer $\alpha$ (at level 0.5) flowing through a pore network of silica micro-spheres. Colors are quantifying the velocity magnitude and the grey isosurface shows the porous medium structure. During this simulation, $\alpha$ is convected through the pore network showing its connectedness. Courtesy of Pore Network Generation Comparison project for the data of body B. (Color online). 
first vector of the standard basis and the second (resp. the third) column with the second (resp. the third) vector of this basis. In this way the fluid velocity can be recomputed easily changing both the driving pressure $\nabla \pi$ and viscosity $\mu$ using only a matrix-vector products [28]. This is particularly interesting for industrial applications, for upscaling (in this case the average of the equation (4.5) is performed on the whole domain), or for modeling the medium heterogeneity (in this case, velocity and pressure in equation (4.5) may be averaged on subdomains).

Since the numerical algorithm to compute the Stokes problem has a quasi-linear computational cost, the implemented solver is a very efficient tool to compute this permeability tensor.

\subsection{Application to biological microfluidics: mucus flow in the lung}

In this section, computations of a mucus film in the human lung are presented. Mucus is a biological fluid which covers bronchial walls in the lungs. It has a very important role to play since it protects bronchus from inhaled agents (pathogens, dust, pollution, ...). In healthy configurations this viscous fluid is constantly moving from distal airways to proximal (trachea) where it is carried away in the stomach: this natural phenomenon is called the mucociliary clearance. When it fails stagnation of mucus leads to the development of pathogens or, on the opposite, if the mucus film is too thin bronchial walls are exposed to the inhaled air. Both these situations lead to serious contaminations.

Mucus is a viscous gel essentially composed of water and mucin proteins. The mucus viscosity is directly linked to the concentration of mucins, which are produced by gobelet cells on the bronchial wall. Aqueous secretions through the bronchial wall tend to transport mucins upward, so the fluid can be divided in two parts. In the neighborhood of the bronchial wall the viscosity is almost equal to water's, while the fluid is much more viscous close to the air-mucus interface. Some authors are considering two distinct fluid layers $[16,39,40]$ : they call the lower one "periciliary fluid layer" and the upper one "mucus layer". The main issue using this modeling is the lack of experimental data to identify a clear interface between both these fluid layers [43]. In fact the transition seems to be continuous as both these fluids are essentially composed of water. Thus the variable viscosity model presented in this paper is a good framework for this simulation, in this case $\alpha$ is this proportion of mucins in the biological fluid. Finally, some authors use non Newtonian constitutive equations, but there is a controversy in the literature between viscoelastic $[34,36,37]$ and viscoplastic $[6,19,31]$ rheologies. Some experimental work remains necessary to discuss this point and the actual variable viscosity model is a good compromise between constant viscosity Newtonian models and controversial non Newtonian models.

On the bronchial wall other cells have a very important role: the epithelium cells. They are composed of cilia, which are immersed in the mucus film. These cilia are beating in a coordinated manner to propel the biological fluid from distal to proximal airways.

As the characteristic dimension of the flow is microfluidics, gravity forces can be neglected with respect to diffusion forces and the RHS cancels: $f=0$. Consequently, in the solid domain (cilia) the mass fraction $\alpha$ does not play any role in the computations since the penalty term dominates the viscous one.

A simulation of this mucus film around epithelium cilia is presented. The mucus film is initialized with a stratified mucin distribution and the mucus motion is the solution to the problem (2.2) where cilium velocity is prescribed using the penalty technique. The motion of cilia (and the associated cilia velocity $\bar{u}$ ) is computed using a 1D convection equation set on a parametric curve with both oscillating convection field and boundary conditions - see [11] for equations details. Motion can be divided in two phases: first a "recovery" phase (when cilia are beating backward: from proximal to distal airways) then an "effective strokes" (the inverse motion) where cilia tips is higher in the film.

Here we present a simulation involving a carpet of cilia. A decay is added to the oscillation to get an asynchronous beating: in a row of cilia, the proximal cilium beats in advance, this phenomenon, observed experimentally [38], is called the methachronal synchronization. It has been studied numerically [26,34] and experimentally [25].

The simulation involves 300 cilia organized as a $100 \times 3$ regular set. The beating motion is dimensionized such that no collisions can occur between adjacent cilia, the beating frequency is $10 \mathrm{~Hz}$. For the discretization $4096 \times 128 \times 256$ grid points are used. The temporal integration is performed with a Runge-Kutta 2 scheme 

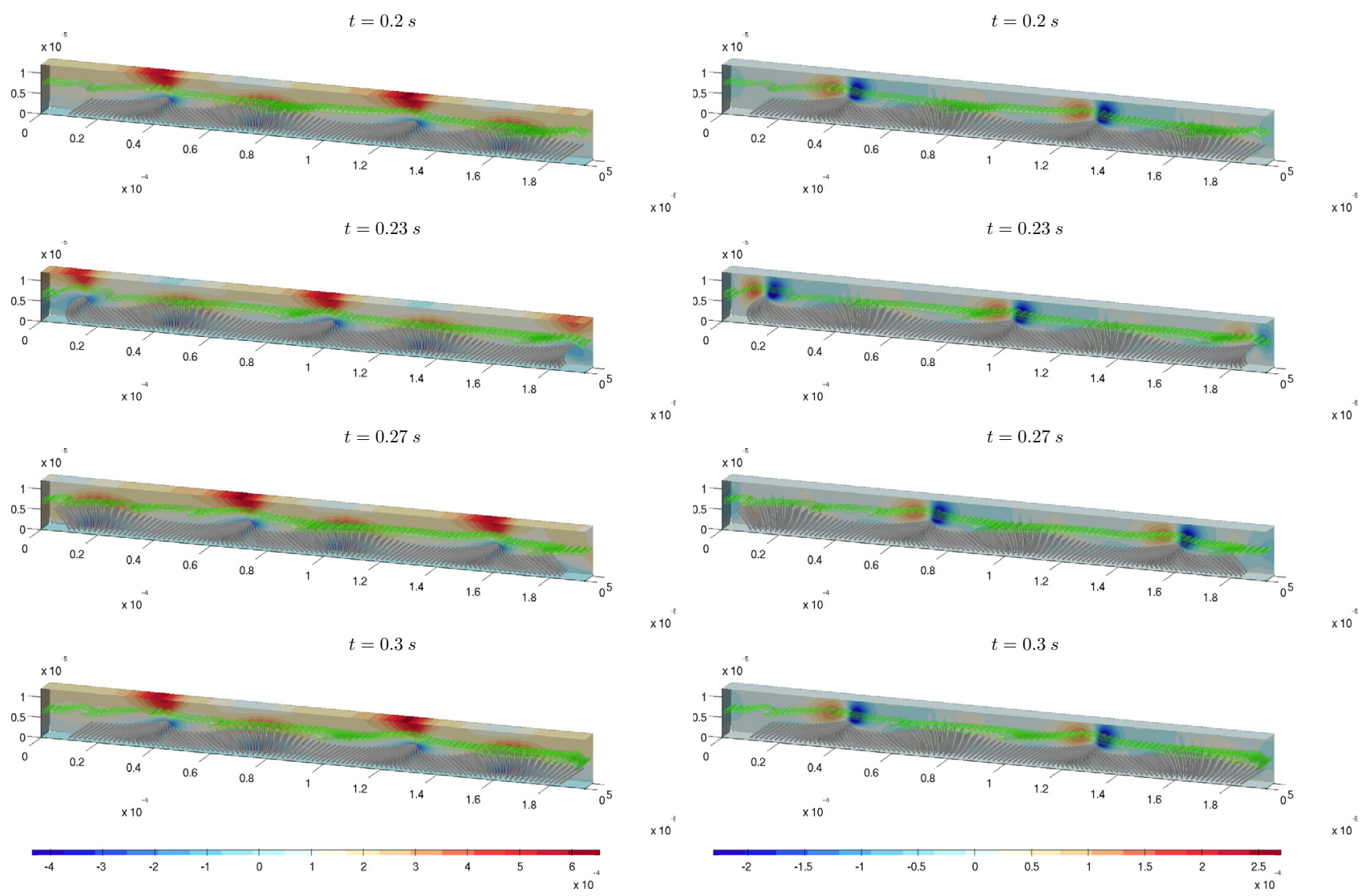

$\times 10^{+}$

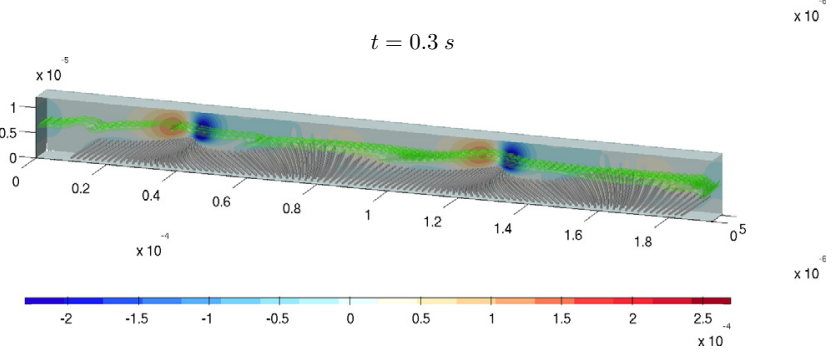

FiguRE 3. Different snapshots of the epithelium cilia carpet beating in a pulmonary mucus at four different instants. On the left, the colormap shows the magnitude of the first component of the velocity field norm, on the right it shows the third component of the velocity field. At the beginning of the computation viscosity is stratified: equal to water's at the bottom $\left(10^{-3} \mathrm{Pas}\right)$ and sixty times more viscous at the top of the computational box. In green, an isosurface of viscosity of level $4 \times 10^{-2}$ Pas is represented. (Color online).

since Adams schemes do not fill very well for Lagrangian computations when the acceleration sign changes. The simulation was performed over ten beating periods and the computational algorithm is able to handle viscosity gradients with robustness, even for large time steps and the computational cost remains quasi-linear [10].

On Figure 3 different snapshots of the simulation are presented at different times, showing the methachronal synchronization of cilia along two wave lengths. In the first (respectively second) column, the colormap quantifies the magnitude of the first (respectively third) component of the velocity field. An isosurface of viscosity, which is deformed by cilia beating, is also printed in green. These pictures clearly show that the methachronal wave travels backward on the cilia carpet, but at the surface of the film, the first component of the velocity remains positive, even where cilia are beating backward. This is the consequence of a "driven cavity" effect which emerges exactly at this location. It is identified with the characteristic double shear stress on both $x$ and $z$ velocity components. This result shows that the methachronal synchronization is an important phenomenon to achieve an efficient mucociliary clearance, since a coordinated beating leads to a negative velocity when cilia beat backward [11]. This result was already observed numerically in 2D [26], with simplified cilia, modeled as 
rigid beating cylinder. The reproduction of this result in a $3 \mathrm{D}$ configuration validates the different methodologies used in [26] and in the present paper.

\section{Conclusion}

This paper focuses on the penalized Stokes problem, modelling a coupling between a highly viscous fluid and a moving obstacle. The composition of this fluid is not homogeneous: its viscosity is a function of the mass fraction of a certain diluted agent. This mass fraction is the solution to a transport-diffusion equation coupled with the fluid velocity, so the dynamics is non linear.

The first main theorem of this paper gives the existence and uniqueness of the solution to this equation system with regularity estimates. The second main theorem shows that the solution to the penalized equation system converges weakly towards the solution to the physical problem as the penalty parameter goes to zero. Two numerical simulations of 3D flow configurations using an algorithm based on this model are also presented.

In the future, a study of the boundary layer created around and inside obstacles, with an asymptotic expansion of the solution, will investigate how both the time dependency and the non-homogeneous fluid influence the well-posedness of the problem and the solution regularity. A second improvement in the model will be to consider a free surface, particularly for investigation of mucociliary clearance: it will require additional theoretical computations and numerical improvements of the computational algorithm. An analysis of the 2-ways coupling (it means to consider the force exerted by the fluid on the obstacles) and its implementation will also complete this work. Finally homogenization techniques will be used on this model to study the behavior of larger cilia carpets.

Acknowledgements. This work is supported by the ANR Grant BioFiReaDy, under the contract number ANR-2010JCJC-0113-01. This work was granted access to the HPC resources of CALMIP under the allocation P0314.

\section{REFERENCES}

[1] J.C. Adams, mudpack: Multigrid portable fortran software for the efficient solution of linear elliptic partial differential equations. Appl. Math. Comput. 34 (1989) 113-146.

[2] R.A. Adams and J.J.F. Fournier, Sobolev Spaces. Academic Press (2003).

[3] S. Agmon, Lectures on Elliptic Boundary Value Problems. Prepared for Publication by B. Frank Jones, with the Assistance of George W. Batten (1965).

[4] Ph. Angot, Analysis of singular perturbations on the brinkman problem for fictitious domain models of viscous flowsh. Math. Methods Appl. Sci. 22 (1999) 1395-1412.

[5] Ph. Angot, Ch.-H. Bruneau and P. Fabrie, A penalization method to take into account obstacles in incompressible viscous flows. Numer. Math. 81 (1999) 497-520.

[6] G.J. Besseris and D.B. Yeates, Rotating magnetic particle microrheometry in biopolymer fluid dynamics: Mucus microrheology. J. Chem. Phys. 127 (2007) 105106-105106.

[7] C. Bost, G.-H. Cottet and E. Maitre, Convergence analysis of a penalization method for the three-dimensional motion of a rigid body in an incompressible viscous fluid. SIAM J. Numer. Anal. 48 (2010) 1313-1337.

[8] F. Boyer and P. Fabrie, Eléments d'analyse pour l'étude de quelques modèles d'écoulements de fluides visqueux incompressibles. Springer (2005).

[9] G. Carbou and P. Fabrie, Boundary layer for a penalization method for viscous incompressible flow. Adv. Differ. Eq. 8 (2003) $1453-1480$.

[10] R. Chatelin, Méthodes numériques pour l'écoulement de Stokes 3D: fluides à viscosité variable en géométrie complexe mobile; application aux fluides biologiques. Ph.D. thesis, Université Toulouse 3 Paul Sabatier (2013).

[11] R. Chatelin and Ph. Poncet, A hybrid grid-particle method for moving bodies in 3D stokes flow with variable viscosity. SIAM J. Sci. Comput. 35 (2013) B925-B949.

[12] R. Chatelin and Ph. Poncet, Hybrid grid-particle methods and penalization: A Sherman-Morrison-Woodbury approach to compute 3D viscous flows using FFT. J. Comput. Phys. 269 (2014) 314-328.

[13] G.-H. Cottet and P. Poncet, Advances in direct numerical simulations of 3D wall-bounded flows by vortex-in-cell methods. J. Comput. Phys. 193 (2004) 136-158.

[14] M. Coquerelle and G.-H. Cottet, A vortex level set method for the two-way coupling of an incompressible fluid with colliding rigid bodies. J. Comput. Phys. 227 (2008) 9121-9137.

[15] G.-H. Cottet and P.D. Koumoutsakos, Vortex Methods: Theory and Practice. Cambridge University Press (2000). 
[16] R.V. Craster and O.K. Matar, Surfactant transport on mucus films. J. Fluid Mech. 425 (2000) 235-258.

[17] R. Dautray and J.L. Lions, Mathematical Analysis and Numerical Methods for Science and Technology. Vol. 1-6. Springer (2000).

[18] G. Duvaut and J.L. Lions, Les inéquations en mécanique et en physique (1972).

[19] P.A. Edwards and D.B. Yeates, Magnetic Rheometry of Bronchial Mucus. In vol. 489 of Viscoelasticity of Biomaterials, ACS Symposium Series. American Chemical Society (1992) 249-267.

[20] M. El Ossmani and P. Poncet, Efficiency of multiscale hybrid grid-particle vortex methods. Multiscale Model. Simul. 8 (2010) 1671-1690.

[21] C. Foias and R. Temam, Remarques sur les équations de navier-stokes stationnaires et les phénomènes successifs de bifurcation. Ann. Sc. Norm. Super. Pisa - Cl. Sci. 5 (1978) 29-63.

[22] R. Glowinski, T.-W. Pan, T.I. Hesla and D.D. Joseph, A distributed lagrange multiplier/fictitious domain method for particulate flows. Int. J. Multiphase Flow 25 (1999) 755-794.

[23] G. Hou, J. Wang and A. Layton, Numerical methods for fluid-structure interaction - a review. Commun. Comput. Phys. 12 (2012) 337-377.

[24] H.H. Hu, Direct simulation of flows of solid-liquid mixtures. Int. J. Multiphase Flow 22 (1996) 335-352.

[25] J. Hussong, N. Schorr, J. Belardi, O. Prucker, J. Rhe and J. Westerweel, Experimental investigation of the flow induced by artificial cilia. Lab on a Chip 11 (2011) 2017-2022.

[26] J. Hussong, W.-P. Breugem and J. Westerweel, A continuum model for flow induced by metachronal coordination between beating cilia. J. Fluid Mech. 684 (2011) 137-162.

[27] J. Janela, A. Lefebvre and B. Maury. A penalty method for the simulation of fluid-rigid body interaction. ESAIM: Procs. 14 (2005) 115-123.

[28] M. Krotkiewski, I.S. Ligaarden, K.-A. Lie and D.W. Schmid, On the importance of the stokes-brinkman equations for computing effective permeability in karst reservoirs. Commun. Comput. Phys. 10 (2011) 1315-1332.

[29] A. Lefebvre, Fluid-particle simulations with FreeFem++. ESAIM: Procs. 18 (2007) 120-132.

[30] J.L. Lions-Magenes, Problèmes aux limites non homogènes et applications. In vol. 1 (1968).

[31] O.K. Matar, R.V. Craster and M.R.E. Warner, Surfactant transport on highly viscous surface films. J. Fluid Mech. 466 (2002) $85-111$.

[32] B. Maury, Direct simulations of 2D fluid-particle flows in biperiodic domains. J. Comput. Phys. 156 (1999) 325-351.

[33] B. Maury, Numerical analysis of a finite element/volume penalty method. SIAM J. Numer. Anal. 47 (2009) $1126-1148$.

[34] S.M. Mitran, Metachronal wave formation in a model of pulmonary cilia. Comput. Struct. 85 (2007) $763-774$.

[35] J. Nečas, Les méthodes directes en théorie des équations elliptiques. Academia (1967).

[36] E. Puchelle, J.M. Zahm and C. Duvivier, Spinability of bronchial mucus. relationship with viscoelasticity and mucous transport properties. Biorheology 20 (1983) 239-249. PMID: 6871438.

[37] E. Puchelle, J.M. Zahm and D. Quemada, Rheological properties controlling mucociliary frequency and respiratory mucus transport. Biorheology 24 (1987) 557-563. PMID: 3502756.

[38] M.J. Sanderson and M.A. Sleigh, Ciliary activity of cultured rabbit tracheal epithelium: beat pattern and metachrony. J. Cell Sci. 47 (1981) 331-347.

[39] D.J. Smith, E.A. Gaffney and J.R. Blake, Modelling mucociliary clearance. Respiratory Phys. Neurobiology 163 (2008) $178-188$.

[40] D.J. Smith, E.A. Gaffney and J.R. Blake. A viscoelastic traction layer model of muco-ciliary transport. Bull. Math. Biology 69 (2007) 289-327.

[41] P. Swarztrauber and R. Sweet, Efficient FORTRAN subprograms for the solution of elliptic partial differential equations (abstract). SIGNUM Newsl. 10 (1975).

[42] R.A. Sweet. A parallel and vector variant of the cyclic reduction algorithm. SIAM J. Sci. Statist. Comput. 9 (1988) $761-765$.

[43] M. Thiriet, Tissue Functioning and Remodeling in the Circulatory and Ventilatory Systems. In vol. 5 in Biomathematical and Biomechanical Modeling of the Circulatory and Ventilatory Systems. Springer, Dordrecht (2012). 